\title{
Efficient Semiparametric Estimation in Generalized Partially Linear Additive Models for Longitudinal/Clustered Data
}

\author{
Guang Cheng*, Lan Zhou ${ }^{\dagger}$ and Jianhua Z. Huang ${ }^{\ddagger}$. \\ Purdue University and Texas AGM University
}

In this supplementary note, we discuss the properties of the least favorable directions, and provide the complete proofs of estimation consistency and asymptotic normality together with some empirical process results for clustered/longitudinal data. We also include some additional numerical results. The note is organized as follows. Section S.1 discusses the properties of the LFD under primitive conditions. Section S.2 defines the inner products and norms to be used in the note. Section S.3 presents the empirical processes tools we develop for the clustered/longitudinal data. Section S.4 contains the estimation consistency proof. Section S.5 collects the lemmas needed for the asymptotic normality proof which is given in Section S.6. Section S.7 reports the results of a simulation study that compares our method with that by Carroll et al. (2009).

\section{S.1. Properties of the least favorable directions}

In this subsection, we show that the least favorable directions (or, more generally, $\varphi_{k d}^{*}\left(t_{d}\right)$ 's) are well defined and have nice properties such as boundedness and smoothness under reasonable assumptions on the joint density of $\mathbf{X}_{i}$ and $\mathbf{T}_{i}$. These properties are crucial for the feasibility to construct semiparametric efficient estimators but are not carefully studied in the literature. We present our results for the generalized efficient score functions, which are defined using the working covariance matrices. Recall that $\varphi_{k,+}^{*}(\mathbf{t})=\varphi_{k 1}^{*}\left(t_{1}\right)+\cdots+$ $\varphi_{k D}^{*}\left(t_{D}\right)$ is the additive function $\varphi_{k,+}(\cdot)$ that minimizes

$$
\sum_{i=1}^{n} E\left[\left\{\boldsymbol{X}_{i k}-\varphi_{k,+}\left(\mathbf{T}_{i}\right)\right\}^{\prime} \boldsymbol{\Delta}_{i 0} \mathbf{V}_{i}^{-1} \boldsymbol{\Delta}_{i 0}\left\{\boldsymbol{X}_{i k}-\varphi_{k,+}\left(\mathbf{T}_{i}\right)\right\}\right]
$$

\footnotetext{
${ }^{*}$ Corresponding author. Purdue University, West Lafayette, IN 47907, Email: chengg@purdue.edu. Research sponsored by NSF (DMS-0906497) and NSF CAREER Award (DMS-1151692).

†Texas A\&M University, College Station, TX 77843, Email: lzhou@stat.tamu.edu. Research partly sponsored by NSF (DMS-0907170)

¥Texas A\&M University, College Station, TX 77843, Email: jianhua@stat.tamu.edu. Research partly sponsored by NSF (DMS-0606580, DMS-0907170), NCI (CA57030), and Award Number KUS-CI-016-04, made by King Abdullah University of Science and Technology (KAUST).
} 
Since $\varphi_{k,+}^{*}(\cdot)$ can be viewed as an orthogonal projection, it always exists and is uniquely defined under the condition that $\mu(\cdot)$ is strictly monotone and $\mathbf{V}_{i}^{-1}$ is positive definite, see the alternating projection theorem (Bickel et al., 1993, Theorem A.4.2).

In general, $\varphi_{k d}^{*}(\cdot)$ 's do not have a closed-form expression. We now show that they are the solution of a Fredholm integral equation of the second kind (Kress, 1999). The stationary equations of the minimization problem are, for $k=1,2 \ldots, K$,

$$
E\left[\sum_{i=1}^{n} \sum_{j, j^{\prime}=1}^{m_{i}}\left\{X_{i j k}-\sum_{d^{\prime}=1}^{D} \varphi_{k d^{\prime}}^{*}\left(T_{i j d^{\prime}}\right)\right\} \Delta_{i j j} v_{i}^{j j^{\prime}} \Delta_{i j^{\prime} j^{\prime}} \varphi_{d}\left(T_{i j^{\prime} d}\right)\right]=0,
$$

for any $\varphi_{d} \in L_{2}\left(\mathcal{T}_{d}\right), d=1, \ldots, D$, where $\Delta_{i j j}$ is the $j$-th diagonal element of the diagonal matrix $\boldsymbol{\Delta}_{i 0}$, and $v_{i}^{j j^{\prime}}$ is the Recall that $f_{i j d}$ and $f_{i j j^{\prime} d d^{\prime}}$ are the density function for $T_{i j d}$ and the joint density function for $\left(T_{i j d}, T_{i j^{\prime} d^{\prime}}\right)$, respectively. By considering conditional expectations and writing out the conditional expectations as integrals, we see that $\varphi_{k d}^{*}(\cdot)$ 's solve the following system of Fredholm integral equations of the second kind (Kress, 1999):

$$
\begin{aligned}
\varphi_{k d}^{*}(t)=q_{d}(t) & -\int_{\mathcal{T}_{d}} H_{d d}(t, s) \varphi_{k d}^{*}(s) d s \\
& -\sum_{d^{\prime} \neq d} \int_{\mathcal{T}_{d^{\prime}}}\left[G_{d d^{\prime}}(t, s)+H_{d d^{\prime}}(t, s)\right] \varphi_{k d^{\prime}}^{*}(s) d s
\end{aligned}
$$

where

$$
q_{d}(t)=\frac{a_{d}(t)}{b_{d}(t)}, \quad H_{d d^{\prime}}(t, s)=\frac{A_{d d^{\prime}}(t, s)}{b_{d}(t)}, \quad G_{d d^{\prime}}(t, s)=\frac{B_{d d^{\prime}}(t, s)}{b_{d}(t)}
$$

with

$$
\begin{aligned}
a_{d}(t) & =\sum_{i} \sum_{j} \sum_{j^{\prime}} E\left(\Delta_{i j j^{\prime}} v_{i}^{j j^{\prime}} \Delta_{i j^{\prime} j^{\prime}} X_{i j k} \mid T_{i j^{\prime} d}=t\right) f_{i j^{\prime} d}(t), \\
b_{d}(t) & =\sum_{i} \sum_{j} E\left(\Delta_{i j j}^{2} v_{i}^{j j} \mid T_{i j d}=t\right) f_{i j d}(t), \\
A_{d d^{\prime}}(t, s) & =\sum_{i} \sum_{j} \sum_{j^{\prime} \neq j} E\left(\Delta_{i j j} v_{i}^{j j^{\prime}} \Delta_{i j^{\prime} j^{\prime}} \mid T_{i j d}=t, T_{i j^{\prime} d^{\prime}}=s\right) f_{i j j^{\prime} d^{\prime} d}(t, s), \\
B_{d d^{\prime}}(t, s) & =\sum_{i} \sum_{j} E\left(\Delta_{i j j}^{2} v_{i}^{j j} \mid T_{i j d}=t, T_{i j d^{\prime}}=s\right) f_{i j j d d^{\prime}}(t, s),
\end{aligned}
$$

for any fixed $d=1,2, \ldots, D$ and $k=1,2, \ldots, K$.

By condition $\mathrm{C} 1, \mathrm{C} 2, \mathrm{C} 4, \mathrm{C} 5$ (iii), and the boundedness of $\theta_{0,+}$, it can be concluded that $A_{d d^{\prime}}(t, s) / n$ and $a_{d}(t) / n$ are bounded, and $b_{d}(t) / n$ is bounded below by some positive constant that is independent of $n$. Consequently, $H_{d d^{\prime}}(t, s)$ and $q_{d}(t)$ are both bounded. Using the fact that $\varphi_{k d}^{*} \in L_{2}\left(\mathcal{T}_{d}\right)$, and applying the Cauchy-Schwarz inequality for the integrals in (S.2), we obtain that $\varphi_{k d}^{*}$ is bounded. 
We next show that the smoothness of $\varphi_{k d}^{*}$ follows from the smoothness assumptions on the joint density of $\mathbf{X}_{i}$ and $\mathbf{T}_{i}$. This result is useful to verify Condition C8'. To prove this result, we prepare with a lemma.

Let $\lceil\alpha\rceil$ denote the smallest integer bigger than or equal to $\alpha$ and $m_{\alpha}=\lceil\alpha\rceil-1$. We say a function $a(\mathbf{s}, t)$ supported on compacta is uniformly $\alpha$-smooth in $t$ relative to $\mathbf{s}$ if the $m$-th derivative of $a(\mathbf{s}, t)$ w.r.t. $t$, denoted as $D_{t}^{m} a(\mathbf{s}, t)$, for $m=0, \ldots, m_{\alpha}$, are all bounded, and

$$
\sup _{\mathbf{s}} \sup _{t_{1} \neq t_{2}} \frac{\left|D_{t}^{m_{\alpha}} a\left(\mathbf{s}, t_{2}\right)-D_{t}^{m_{\alpha}} a\left(\mathbf{s}, t_{1}\right)\right|}{\left|t_{2}-t_{1}\right|^{\alpha^{\prime}}}<\infty
$$

with $\alpha^{\prime}=\alpha-m_{\alpha}$.

LEMma S.1. (i) If $a(\mathbf{s}, t)=a\left(\mathbf{s}_{1}, \mathbf{s}_{2}, t\right)$ is uniformly $\alpha$-smooth in $t$ relative to $\mathbf{s}_{1}$ and $\mathbf{s}_{2}$, then $\int_{\mathcal{S}_{1}} a\left(\mathbf{s}_{1}, \mathbf{s}_{2}, t\right) d \mathbf{s}_{1}$ is uniformly $\alpha$-smooth in $t$ relative to $\mathbf{s}_{2}$.

(ii) If $a(\mathbf{s}, t)$ and $b(\mathbf{s}, t)$ are both uniformly $\alpha$-smooth in $t$ relative to $\mathbf{s}$, then $c(\mathbf{s}, t) \equiv$ $a(\mathbf{s}, t) b(\mathbf{s}, t)$ is uniformly $\alpha$-smooth in $t$ relative to $\mathbf{s}$.

(iii) If $a(\mathbf{s}, t)$ is uniformly $\alpha$-smooth in $t$ relative to $\mathbf{s}$ and $f(\cdot) \in C^{\lceil\alpha\rceil}$, then $f(a(\mathbf{s}, t))$ is uniformly $\alpha$-smooth in $t$ relative to $\mathbf{s}$.

Proof: (i) Note that $D_{t}^{m} a\left(\mathbf{s}_{1}, \mathbf{s}_{2}, t\right)$ is bounded for $0 \leq m \leq m_{\alpha}$, by the dominated convergence theorem, we can take derivative inside the integral to obtain

$$
D_{t}^{m}\left(\int_{\mathcal{S}_{1}} a\left(\mathbf{s}_{1}, \mathbf{s}_{2}, t\right) d \mathbf{s}_{1}\right)=\int_{\mathcal{S}_{1}} D_{t}^{m} a\left(\mathbf{s}_{1}, \mathbf{s}_{2}, t\right) d \mathbf{s}_{1}
$$

which implies that $D_{t}^{m}\left(\int_{\mathcal{S}_{1}} a\left(\mathbf{s}_{1}, \mathbf{s}_{2}, t\right) d \mathbf{s}_{1}\right)$ is bounded for $0 \leq m \leq m_{\alpha}$. Using this and the fact that

$$
\begin{aligned}
& \frac{\left|D_{t}^{m_{\alpha}}\left(\int_{\mathcal{S}_{1}} a\left(\mathbf{s}_{1}, \mathbf{s}_{2}, t_{2}\right) d \mathbf{s}_{1}\right)-D_{t}^{m_{\alpha}}\left(\int_{\mathcal{S}_{1}} a\left(\mathbf{s}_{1}, \mathbf{s}_{2}, t_{1}\right) d \mathbf{s}_{1}\right)\right|}{\left|t_{2}-t_{1}\right|^{\alpha^{\prime}}} \\
& \leq \int_{\mathcal{S}_{1}} \sup _{\mathbf{s}_{1}, \mathbf{s}_{2}} \sup _{t_{1} \neq t_{2}} \frac{\left|D_{t}^{m_{\alpha}} a\left(\mathbf{s}_{1}, \mathbf{s}_{2}, t_{2}\right)-D_{t}^{m_{\alpha}} a\left(\mathbf{s}_{1}, \mathbf{s}_{2}, t_{1}\right)\right|}{\left|t_{2}-t_{1}\right|^{\alpha^{\prime}}} d \mathbf{s}_{1}<\infty,
\end{aligned}
$$

for all $\mathbf{s}_{2}$ and $t_{1} \neq t_{2}$, we conclude that $\int_{\mathcal{S}_{1}} a\left(\mathbf{s}_{1}, \mathbf{s}_{2}, t\right) d \mathbf{s}_{1}$ is indeed uniformly $\alpha$-smooth in $t$ relative to $\mathbf{s}_{2}$.

(ii) The result is true because

$$
D_{t}^{m} c=\sum_{i+j=m} D_{t}^{i} a D_{t}^{j} b
$$

is bounded for $0 \leq m \leq m_{\alpha}$. Also we note that for $i<m_{\alpha}$,

$$
\frac{\left|D_{t}^{i} a\left(\mathbf{s}, t_{2}\right)-D_{t}^{i} a\left(\mathbf{s}, t_{1}\right)\right|}{\left|t_{2}-t_{1}\right|^{\alpha^{\prime}}}=\frac{\left|\int_{t_{1}}^{t_{2}} D_{t}^{i+1} a(\mathbf{s}, t) d t\right|}{\left|t_{2}-t_{1}\right|^{\alpha^{\prime}}} .
$$


It can then be easily verified that

$$
\sup _{\mathbf{s}} \sup _{t_{1} \neq t_{2}} \frac{\left|D_{t}^{m_{\alpha}} c\left(\mathbf{s}, t_{2}\right)-D_{t}^{m_{\alpha}} c\left(\mathbf{s}, t_{1}\right)\right|}{\left|t_{2}-t_{1}\right|^{\alpha^{\prime}}}<\infty .
$$

(iii) When $0<\alpha \leq 1$, the result follows from the observation that

$$
\frac{f\left(a\left(\mathbf{s}, t_{2}\right)\right)-f\left(a\left(\mathbf{s}, t_{1}\right)\right)}{\left|t_{2}-t_{1}\right|^{\alpha}}=\frac{f\left(a\left(\mathbf{s}, t_{2}\right)\right)-f\left(a\left(\mathbf{s}, t_{1}\right)\right)}{\left|a\left(\mathbf{s}, t_{2}\right)-a\left(\mathbf{s}, t_{1}\right)\right|} \cdot \frac{\left|a\left(\mathbf{s}, t_{2}\right)-a\left(\mathbf{s}, t_{1}\right)\right|}{\left|t_{2}-t_{1}\right|^{\alpha}} .
$$

Using the chain rule, the above observation and part (ii) of the lemma, the desired result can be obtained by induction for general $\alpha$.

Note that $\varphi_{k d}^{*}$ 's satisfy the system of integral equations (S.2). The quantities involved in the integral equations have certain properties under the regularity conditions listed in Section 3.1 Under Condition C5(ii) $\mu(\cdot)$ is strictly monotone. Condition C4 states that $\mathbf{V}_{i}^{-1}$ is positive definite. It follows that $b_{d}(t)$ is strictly positive. According to Lemma S.1, we have that $q_{d}(t), \int_{\mathcal{T}_{d^{\prime}}} H_{d d^{\prime}}(t, s) \varphi_{k d^{\prime}}^{*}(s) d s$ and $\int_{\mathcal{T}_{d^{\prime}}} G_{r d^{\prime}}(t, s) \varphi_{k d^{\prime}}^{*}(s) d s$ are $\alpha$-smooth in $t$ if we assume that $f_{X, T}\left(\mathbf{x}_{i}, \mathbf{t}_{i}\right)$ and elements of $\mathbf{V}_{i}^{-1}=\left(v_{i}^{k l}\right)$ are all uniformly $\alpha$-smooth in $t_{i j d}$ relative to the rest of the variates, and also that $\mu^{\prime}(\cdot) \in C^{\lceil\alpha\rceil}$. Therefore, it follows from (S.2) that $\varphi_{k d}^{*}$ is $\alpha$-smooth.

\section{S.2. Inner products and norms}

Our theoretical development extends the geometric arguments used in Huang (1998, 2003), and Huang, Wu and Zhou (2004). This subsection gives the definition of some relevant inner products and norms in our context and presents some useful lemmas. We first introduce two inner products. For $\xi_{1}, \xi_{2} \in L_{2}(d \mathbf{x} \times d \mathbf{t})$, define the empirical inner product as

$$
\left\langle\xi_{1}, \xi_{2}\right\rangle_{n}^{\Delta}=\frac{1}{n} \sum_{i} \xi_{1}^{\prime}\left(\mathbf{X}_{i}, \mathbf{T}_{i}\right) \boldsymbol{\Delta}_{i 0} \mathbf{V}_{i}^{-1} \boldsymbol{\Delta}_{i 0} \xi_{2}\left(\mathbf{X}_{i}, \mathbf{T}_{i}\right)
$$

and the theoretical inner product as $\left\langle\xi_{1}, \xi_{2}\right\rangle^{\Delta}=E\left[\left\langle\xi_{1}, \xi_{2}\right\rangle_{n}^{\Delta}\right]$. Corresponding norms are denoted as $\|\cdot\|_{n}^{\Delta}$ and $\|\cdot\|^{\Delta}$. Note that the superscript $\Delta$ reflects the dependence on the $\Delta_{i 0}$. When the link function is the identity function, two $\Delta_{i 0}$ 's become the identity matrix and thus $\langle\cdot, \cdot\rangle_{n}^{\Delta}$ reduced to $\langle\cdot, \cdot\rangle_{n}$ as previously defined in Section 3.3 of the main paper.

Lemma S.2. (i) There are constants $M_{1}, M_{2}>0$ such that $M_{1}\|g\|_{L_{2}} \leq\|g\|^{\Delta} \leq M_{2}\|g\|_{L_{2}}$ for all $g \in \mathbb{G}_{+}$. (ii) There is a constant $M_{3}$ such that $\|g\|_{\infty} \leq M_{3} Q_{n}^{1 / 2}\|g\|^{\Delta}$ for all $g \in \mathbb{G}_{+}$. (iii) There is a constant $M_{4}$ such that $\left\|\mathbf{x}^{\prime} \beta+g(\mathbf{t})\right\|_{\infty} \leq M_{4} Q_{n}{ }^{1 / 2}\left\|\mathbf{x}^{\prime} \beta+g(\mathbf{t})\right\|$ for $g \in \mathbb{G}_{+}$.

Let $x_{k}(\cdot)$ denote the coordinate mapping that maps $x$ to its $k$-th component so that $x_{k}\left(X_{i j}\right)=X_{i j k}$ for $k=1, \ldots, p$. 
Lemma S.3. Suppose $\lim _{n} Q_{n}^{2} \log n / n=0$. Then, for $k=1, \ldots, p$,

$$
\begin{aligned}
\sup _{g \in \mathbb{G}_{+}}\left|\frac{\left[\left\|x_{k}-g\right\|_{n}^{\Delta}\right]^{2}}{\left[\left\|x_{k}-g\right\|^{\Delta}\right]^{2}}-1\right| & =o_{P}(1), \\
\sup _{g \in \mathbb{G}_{+}}\left|\frac{\left[\|g\|_{n}^{\Delta}\right]^{2}}{\left[\|g\|^{\Delta}\right]^{2}}-1\right| & =o_{P}(1) .
\end{aligned}
$$

Lemma S.4. Let $\left\{h_{n}\right\}$ be a sequence of functions on $\mathcal{T}$ such that $\left\|h_{n}\right\|_{\infty} \leq M$ for $n \geq 1$. Then,

$$
\sup _{g \in \mathbb{G}_{+}} \frac{\left|\left\langle h_{n}, g\right\rangle_{n}^{\Delta}-\left\langle h_{n}, g\right\rangle^{\Delta}\right|}{\|g\|^{\Delta}}=O_{P}\left(\sqrt{\frac{Q_{n}}{n}}\right)\left\|h_{n}\right\|^{\Delta} .
$$

These lemmas are slight extensions of existing results. Lemma S.2 follows from Conditions C1 and C5 (ii)-(iii), Lemma 3.1 of Stone (1994), and a property of the spline functions as presented in Appendix A2 of Huang, Wu and Zhou (2004). Lemma S.3 can be proved as Lemma A2 of Huang, Wu and Zhou (2004). Lemma S.4 follows from the same argument as the proof of Lemma 11 in Huang (1998).

\section{S.3. Empirical process results for clustered/longitudinal data}

To facilitate our asymptotic analysis, we provide in this section some empirical process results for clustered/longitudinal data, which are extensions of existing results for i.i.d. data (e.g., Chapters 8 \& 9 of van de Geer, 2000).

Lemma S.5. Let $\boldsymbol{W}=\left(W_{1}, \ldots, W_{m}\right)^{\prime}$ be a zero mean random vector that satisfies the sub-Gaussian condition:

$$
M_{0}^{2}\left\{E \exp \left(|\boldsymbol{W}|^{2} / M_{0}^{2}\right)-1\right\} \leq \sigma_{0}^{2},
$$

Let $\mathbf{V}$ be a symmetric positive definite matrix. Then, for all $\boldsymbol{\beta} \in \mathbb{R}^{m}$,

$$
E \exp \left(\boldsymbol{\beta}^{\prime} \mathbf{V}^{-1} \boldsymbol{W}\right) \leq \exp \left\{2\left(M_{0}^{2}+\sigma_{0}^{2}\right)\left|\lambda_{\mathbf{V}^{-1}}^{\max }\right|^{2}|\boldsymbol{\beta}|^{2}\right\} .
$$

Proof. The proof is a slight modification of the proof of Lemma 8.1 on Page 126 of van de Geer (2000). Take $c=1+\sigma_{0}^{2} / M_{0}^{2}$. By (S.5) and Chebyshev's inequality, we have that, for all $t>0$

$$
P(|\boldsymbol{W}|>t) \leq c \exp \left(-t^{2} / M_{0}^{2}\right)
$$

We then have that, for $k=2,3, \ldots$,

$$
E|\boldsymbol{W}|^{k}=\int_{0}^{\infty} k t^{k-1} P(|\boldsymbol{W}|>t) d t \leq c \int_{0}^{\infty} k t^{k-1} \exp \left(-t^{2} / M_{0}^{2}\right) d t
$$


Do the substitution $u=\left(t / M_{0}\right)^{2}$ in the above integral, then $t=M_{0} \sqrt{u}, d t=M_{0} u^{-1 / 2} d u / 2$, and it follows that

$$
E|\boldsymbol{W}|^{k} \leq \frac{c k M_{0}^{k}}{2} \int_{0}^{\infty} u^{k / 2-1} e^{-u} d u=c M_{0}^{k} \Gamma\left(\frac{k}{2}+1\right) .
$$

Note that $E \boldsymbol{\beta}^{\prime} \mathbf{V}^{-1} \boldsymbol{W}=0$ and $\boldsymbol{\beta}^{\prime} \mathbf{V}^{-1} \boldsymbol{W} \leq \lambda_{\mathbf{V}^{-1}}^{\max }|\boldsymbol{\beta} \| \boldsymbol{W}|$. Hence,

$$
\begin{aligned}
E \exp \left\{\boldsymbol{\beta}^{\prime} \mathbf{V}^{-1} \boldsymbol{W}\right\} & \leq 1+\sum_{k=2}^{\infty} \frac{\left(\lambda_{\mathbf{V}^{-1}}^{\max }\right)^{k}|\boldsymbol{\beta}|^{k} E|\boldsymbol{W}|^{k}}{k !} \\
& \leq 1+c \sum_{k=2}^{\infty} \frac{\left(M_{0} \lambda_{\mathbf{V}^{-1}}^{\max }|\boldsymbol{\beta}|\right)^{k}}{k !} \Gamma\left(\frac{k}{2}+1\right) .
\end{aligned}
$$

The fact $\{\Gamma(k / 2+1)\}^{2} \leq \Gamma(k+1)=k !, k=2,3, \ldots$, implies that the above summation is bounded by

$$
\sum_{k=2}^{\infty} \frac{\left(M_{0}|\boldsymbol{\beta}| \lambda_{\mathbf{V}^{-1}}^{\max }\right)^{k}}{\Gamma\left(\frac{k}{2}+1\right)}=\sum_{k=1}^{\infty} \frac{\left\{M_{0}^{2}\left(\lambda_{\mathbf{V}^{-1}}^{\max }\right)^{2}|\boldsymbol{\beta}|^{2}\right\}^{k}}{\Gamma(k+1)}+\sum_{k=1}^{\infty} \frac{\left\{M_{0}^{2}\left(\lambda_{\mathbf{V}^{-1}}^{\max }\right)^{2}|\boldsymbol{\beta}|^{2}\right\}^{k+\frac{1}{2}}}{\Gamma\left(k+\frac{3}{2}\right)}
$$

By induction and the fact that $\Gamma(\ell+1)=\ell \Gamma(\ell), \ell>0$, we can verify that $\Gamma\left(k+\frac{3}{2}\right) \geq$ $\Gamma(k+1), k=1,2, \ldots$ In addition, note that $c<c^{k}$ for $k=2,3 \ldots$ Consequently,

$$
\begin{aligned}
E \exp \left(\boldsymbol{\beta}^{\prime} \mathbf{V}^{-1} \boldsymbol{W}\right) & \leq 1+\sum_{k=1}^{\infty} \frac{\left\{c M_{0}^{2}\left(\lambda_{\mathbf{V}^{-1}}^{\max }\right)^{2}|\boldsymbol{\beta}|^{2}\right\}^{k}}{\Gamma(k+1)}\left[1+\left\{c M_{0}^{2}\left(\lambda_{\mathbf{V}^{-1}}^{\max }\right)^{2}|\boldsymbol{\beta}|^{2}\right\}^{1 / 2}\right] \\
& \leq \exp \left\{c M_{0}^{2}\left(\lambda_{\mathbf{V}^{-1}}^{\max }\right)^{2}|\boldsymbol{\beta}|^{2}\right\}\left(1+\sum_{k=1}^{\infty} \frac{\left\{c M_{0}^{2}\left(\lambda_{\mathbf{V}^{-1}}^{\max }\right)^{2}|\boldsymbol{\beta}|^{2}\right\}^{k}}{\Gamma(k+1)}\right) \\
& \leq \exp \left\{2 c M_{0}^{2}\left(\lambda_{\mathbf{V}^{-1}}^{\max }\right)^{2}|\boldsymbol{\beta}|^{2}\right\}=\exp \left\{2\left(M_{0}^{2}+\sigma_{0}^{2}\right)\left|\lambda_{\mathbf{V}^{-1}}^{\max }\right|^{2}|\boldsymbol{\beta}|^{2}\right\}
\end{aligned}
$$

where the second inequality follows from $A^{1 / 2} \leq e^{A}$ for any $A>0$.

Lemma S.6. Let $\boldsymbol{W}_{1} \in \mathbb{R}^{m_{1}}, \ldots, \boldsymbol{W}_{n} \in \mathbb{R}^{m_{n}}$ be independent zero mean random vectors satisfying the uniform sub-Gaussian condition:

$$
\max _{i=1, \ldots, n} M_{0}^{2}\left\{E \exp \left(\left|\boldsymbol{W}_{i}\right|^{2} / M_{0}^{2}\right)-1\right\} \leq \sigma_{0}^{2} .
$$

Let $\mathbf{V}_{i}^{-1}$ be a symmetric $m_{i} \times m_{i}$ positive definite matrix, $i=1, \ldots, n$. Then, for any $\gamma_{1} \in \mathbb{R}^{m_{1}}, \ldots, \gamma_{n} \in \mathbb{R}^{m_{n}}$, and $a>0$,

$$
P\left(\left|\sum_{i=1}^{n} \boldsymbol{\gamma}_{i}^{\prime} \mathbf{V}_{i}^{-1} \boldsymbol{W}_{i}\right| \geq a\right) \leq 2 \exp \left\{-\frac{a^{2}}{8\left(M_{0}^{2}+\sigma_{0}^{2}\right) \sum_{i=1}^{n}\left(\lambda_{\mathbf{V}_{i}^{-1}}^{\max }\right)^{2}\left|\boldsymbol{\gamma}_{i}\right|^{2}}\right\}
$$


Proof. The proof is almost exactly the same as Lemma 8.2 on Page 127 of van de Geer (2000). We still present it here for completeness. By Lemma S.5, for all $\beta \in \mathbb{R}$,

$$
\begin{aligned}
E \exp \left(\beta \sum_{i=1}^{n} \boldsymbol{\gamma}_{i}^{\prime} \mathbf{V}_{i}^{-1} \boldsymbol{W}_{i}\right) & =\prod_{i=1}^{n} E \exp \left(\beta \boldsymbol{\gamma}_{i}^{\prime} \mathbf{V}_{i}^{-1} \boldsymbol{W}_{i}\right) \\
& \leq \exp \left\{2\left(M_{0}^{2}+\sigma_{0}^{2}\right) \beta^{2} \sum_{i=1}^{n}\left(\lambda_{\mathbf{V}_{i}^{-1}}^{\max }\right)^{2}\left|\boldsymbol{\gamma}_{i}\right|^{2}\right\}
\end{aligned}
$$

This together with the Chebyshev's inequality implies that, for $\beta>0$,

$$
P\left(\sum_{i=1}^{n} \boldsymbol{\gamma}_{i}^{\prime} \mathbf{V}_{i}^{-1} \boldsymbol{W}_{i} \geq a\right) \leq \exp \left\{2\left(M_{0}^{2}+\sigma_{0}^{2}\right) \beta^{2} \sum_{i=1}^{n}\left(\lambda_{\mathbf{V}_{i}^{-1}}^{\max }\right)^{2}\left|\boldsymbol{\gamma}_{i}\right|^{2}-\beta a\right\} .
$$

Take

$$
\beta=\frac{a}{4\left(M_{0}^{2}+\sigma_{0}^{2}\right) \sum_{i=1}^{n}\left(\lambda_{\mathbf{V}_{i}^{-1}}^{\max }\right)^{2}\left|\gamma_{i}\right|^{2}},
$$

in the above inequality to obtain that

$$
P\left(\sum_{i=1}^{n} \boldsymbol{\gamma}_{i}^{\prime} \mathbf{V}_{i}^{-1} \boldsymbol{W}_{i} \geq a\right) \leq \exp \left\{-\frac{a^{2}}{8\left(M_{0}^{2}+\sigma_{0}^{2}\right) \sum_{i=1}^{n}\left(\lambda_{\mathbf{V}_{i}^{-1}}^{\max }\right)^{2}\left|\boldsymbol{\gamma}_{i}\right|^{2}}\right\} .
$$

Because the same inequality holds for $-\sum_{i=1}^{n} \boldsymbol{\gamma}_{i}^{\prime} \mathbf{V}_{i}^{-1} \boldsymbol{W}_{i}$, the desired result follows.

Recall that $\mathbb{F}_{n}=\left\{f(\mathbf{x}, \mathbf{t}): f(\mathbf{x}, \mathbf{t})=\mu\left(\mathbf{x}^{\prime} \boldsymbol{\beta}+g(\mathbf{t})\right), \beta \in \mathbb{R}^{K}, g \in \mathbb{G}_{+}\right\}$. Recall the definition of the norm $\|\cdot\|_{n}$ given above the statement of Theorem 1 . For $R>0$, denote $\mathbb{F}_{n}(R)=\left\{f \in \mathbb{F}_{n}:\left\|f-f_{n}^{*}\right\|_{n} \leq R\right\}$. For $f \in \mathbb{F}_{n}(R), f\left(\mathbf{X}_{i}, \mathbf{T}_{i}\right)$ denotes a vector whose elements are $f\left(\boldsymbol{X}_{i j}, \boldsymbol{T}_{i j}\right), j=1, \ldots, m_{i}$. Let $N\left(r, \mathbb{F}_{n}(R),\|\cdot\|_{n}\right)$ be the minimal number of balls of radius $r$ that is needed to cover $\mathbb{F}_{n}(R)$ under the norm $\|\cdot\|_{n}$. Correspondingly, $H\left(r, \mathbb{F}_{n}(R),\|\cdot\|_{n}\right)=\log N\left(r, \mathbb{F}_{n}(R),\|\cdot\|_{n}\right)$ is the entropy number.

Lemma S.7. Suppose that $\boldsymbol{e}_{i}$ satisfies (10) in the main paper. For some constant $C_{0}$ depending only on $M_{0}$ and $\sigma_{0}$ specified in (10) of the main paper, and for $\delta>0$ and $\sigma>0$ satisfying $\delta / \sigma<R$, and

$$
n^{1 / 2} \delta \geq 2 C_{0}\left\{\int_{\delta / 8 \sigma}^{R} H^{1 / 2}\left(u, \mathbb{F}_{n}(R),\|\cdot\|_{n}\right) d u \bigvee R\right\}
$$

we have that

$$
\begin{gathered}
P\left[\left\{\sup _{f \in \mathbb{F}_{n}(R)}\left|\frac{1}{n} \sum_{i=1}^{n}\left\{f\left(\mathbf{X}_{i}, \mathbf{T}_{i}\right)\right\}^{\prime} \mathbf{V}_{i}^{-1} \boldsymbol{e}_{i}\right| \geq \delta\right\} \cap\left\{\|\boldsymbol{e}\|_{n} \leq \sigma\right\} \mid\left\{\mathbf{X}_{i}, \mathbf{T}_{i}\right\}_{i=1}^{n}\right] \\
\leq C_{0} \exp \left(-\frac{n \delta^{2}}{4 C_{0}^{2} R^{2}}\right) \quad \text { a.s. }
\end{gathered}
$$

where $\boldsymbol{e}=\left(\boldsymbol{e}_{1}^{\prime}, \ldots, \boldsymbol{e}_{n}^{\prime}\right)^{\prime}$ and $\|\boldsymbol{e}\|_{n}^{2}=(1 / n) \sum_{i=1}^{n} \boldsymbol{e}_{i}^{\prime} \mathbf{V}_{i}^{-1} \boldsymbol{e}_{i}$ 
Proof. This is a generalization of Corollary 8.3 of van de Geer (2000) to cluster/longitudinal data. The proof is similar as that of Lemma 3.2 on Page 29 of van de Geer (2000) and included here for completeness. Denote $C^{2}=8 r_{v}\left(M_{0}^{2}+\sigma_{0}^{2}\right)$, where $r_{v}=\max _{1 \leq i \leq n}\left\{\left(\lambda_{\mathbf{V}_{i}^{-1}}^{\max }\right)^{2} / \lambda_{\mathbf{V}_{i}^{-1}}^{\min }\right\}$. According to condition (C4), $r_{v}$ is bounded. By the fact that

$$
\sum_{i=1}^{n}\left|\lambda_{\mathbf{V}_{i}^{-1}}^{\max } \gamma_{i}\right|^{2} \leq r_{v} \sum_{i=1}^{n} \gamma_{i}^{\prime} \mathbf{V}_{i}^{-1} \gamma_{i}:=n r_{v}\|\gamma\|_{n}^{2}
$$

and Lemma S.6, we have

$$
P\left(\frac{1}{n}\left|\sum_{i=1}^{n} \boldsymbol{\gamma}_{i}^{\prime} \mathbf{V}_{i}^{-1} \boldsymbol{e}_{i}\right| \geq a\right) \leq 2 \exp \left\{-\frac{n a^{2}}{C^{2}\|\boldsymbol{\gamma}\|_{n}^{2}}\right\}
$$

To apply the chaining technique, we index the functions in $\mathbb{F}_{n}(R)$ by a parameter $\phi \in \Phi$, i.e. $\mathbb{F}_{n}(R)=\left\{f_{\phi}\right\}_{\phi \in \Phi}$, without loss of generality. Let $N_{s}=N\left(2^{-s} R, \mathbb{F}_{n}(R),\|\cdot\|_{n}\right)$ and $\left\{f_{j}^{s}\right\}_{j=1}^{N_{s}}$ be the minimal $\left(2^{-s} R\right)$-covering set of $\mathbb{F}_{n}(R)$. Then, for each $\phi$, there exists a $f_{\phi}^{s} \in\left\{f_{1}^{s}, \ldots, f_{N_{s}}^{s}\right\}$ such that $\left\|f_{\phi}-f_{\phi}^{s}\right\| \leq 2^{-s} R$. Let

$$
S=\min \left\{s \geq 1: 2^{-s} R \leq \delta / 2 \sigma\right\} .
$$

Note that $S \geq 1$ since we assume $\delta / \sigma<R$. On the set $\left\{\|\boldsymbol{e}\|_{n} \leq \sigma\right\}$, we apply the CauchySchwarz inequality to obtain that

$$
\left|\frac{1}{n} \sum_{i=1}^{n}\left\{f_{\phi}\left(\mathbf{X}_{i}, \mathbf{T}_{i}\right)-f_{\phi}^{S}\left(\mathbf{X}_{i}, \mathbf{T}_{i}\right)\right\}^{\prime} \mathbf{V}_{i}^{-1} \boldsymbol{e}_{i}\right| \leq \sigma\left\|f_{\phi}-f_{\phi}^{S}\right\|_{n} \leq \frac{\delta}{2}
$$

where the second inequality uses (S.12). Then it suffices to prove an analog of (S.10) for the $\left(2^{-S} R\right)$-covering net $\left\{f_{j}^{S}\right\}_{j=1}^{N_{S}}$,

$$
P\left(\max _{j=1, \ldots, N_{S}}\left|\frac{1}{n} \sum_{i=1}^{n}\left\{f_{j}^{S}\left(\mathbf{X}_{i}, \mathbf{T}_{i}\right)\right\}^{\prime} \mathbf{V}_{i}^{-1} \boldsymbol{e}_{i}\right| \geq \frac{\delta}{2} \mid\left\{\mathbf{X}_{i}, \mathbf{T}_{i}\right\}_{i=1}^{n}\right) .
$$

Now apply the chaining technique. Let $f_{\phi}^{0}=0$. We have

$$
f_{\phi}^{S}=\sum_{s=1}^{S}\left(f_{\phi}^{s}-f_{\phi}^{s-1}\right)
$$

By the triangle inequality,

$$
\begin{aligned}
\left\|f_{\phi}^{s}-f_{\phi}^{s-1}\right\|_{n} & \leq\left\|f_{\phi}^{s}-f_{\phi}\right\|_{n}+\left\|f_{\phi}-f_{\phi}^{s-1}\right\|_{n} \\
& \leq 2^{-s} R+2^{-s+1} R=\left(2^{-s}\right) 3 R . \\
& 8
\end{aligned}
$$


Let $\eta_{s}$ be positive numbers satisfying $\sum_{s=1}^{S} \eta_{s} \leq 1$. Then

$$
\begin{aligned}
& P\left(\sup _{\phi \in \Phi}\left|\frac{1}{n} \sum_{s=1}^{S} \sum_{i=1}^{n} \boldsymbol{e}_{i}^{\prime} \mathbf{V}_{i}^{-1}\left\{f_{\phi}^{s}\left(\mathbf{X}_{i}, \mathbf{T}_{i}\right)-f_{\phi}^{s-1}\left(\mathbf{X}_{i}, \mathbf{T}_{i}\right)\right\}\right|>\frac{\delta}{2} \mid\left\{\mathbf{X}_{i}, \mathbf{T}_{i}\right\}_{i=1}^{n}\right) \\
& \leq \sum_{s=1}^{S} P\left(\sup _{\phi \in \Phi}\left|\frac{1}{n} \sum_{i=1}^{n} \boldsymbol{e}_{i}^{\prime} \mathbf{V}_{i}^{-1}\left\{f_{\phi}^{s}\left(\mathbf{X}_{i}, \mathbf{T}_{i}\right)-f_{\phi}^{s-1}\left(\mathbf{X}_{i}, \mathbf{T}_{i}\right)\right\}\right|>\frac{\delta \eta_{s}}{2} \mid\left\{\mathbf{X}_{i}, \mathbf{T}_{i}\right\}_{i=1}^{n}\right) \\
& \leq \sum_{s=1}^{S} 2 \exp \left\{2 H\left(2^{-s} R, \mathbb{F}_{n}(R),\|\cdot\|_{n}\right)-\frac{n \delta^{2} \eta_{s}^{2}}{36 C^{2} 2^{-2 s} R^{2}}\right\}
\end{aligned}
$$

with the last inequality being obtained by the fact that

$$
\begin{aligned}
& \left\{\sup _{\phi \in \Phi}\left|\frac{1}{n} \sum_{i=1}^{n} \boldsymbol{e}_{i}^{\prime} \mathbf{V}_{i}^{-1}\left\{f_{\phi}^{s}\left(\mathbf{X}_{i}, \mathbf{T}_{i}\right)-f_{\phi}^{s-1}\left(\mathbf{X}_{i}, \mathbf{T}_{i}\right)\right\}\right|>\frac{\delta \eta_{s}}{2}\right\} \\
& \subset \bigcup_{1 \leq j \leq N_{s}, 1 \leq l \leq N_{s-1}}\left\{\left|\frac{1}{n} \sum_{i=1}^{n} \boldsymbol{e}_{i}^{\prime} \mathbf{V}_{i}^{-1}\left\{f_{j}^{s}\left(\mathbf{X}_{i}, \mathbf{T}_{i}\right)-f_{l}^{s-1}\left(\mathbf{X}_{i}, \mathbf{T}_{i}\right)\right\}\right|>\frac{\delta \eta_{s}}{2}\right\}
\end{aligned}
$$

and (S.11) and (S.13).

Set

$$
\eta_{s}=\frac{12 C 2^{-s} R H^{1 / 2}\left(2^{-s} R, \mathbb{F}_{n}(R),\|\cdot\|_{n}\right)}{n^{1 / 2} \delta} \bigvee \frac{2^{-s} s^{1 / 2}}{8}
$$

We next verify that $\sum_{s=1}^{S} \eta_{s} \leq 1$. By taking $C_{0}$ sufficiently large in (S.9), we have that

$$
\frac{n^{1 / 2} \delta}{2} \geq 12 C \sum_{s=1}^{S} 2^{-s} R H^{1 / 2}\left(2^{-s} R, \mathbb{F}_{n}(R),\|\cdot\|_{n}\right)
$$

Moreover,

$$
\begin{aligned}
\sum_{s=1}^{S} 2^{-s} s^{1 / 2} & \leq 1+\int_{1}^{\infty} 2^{-x} x^{1 / 2} d x \\
& \leq 1+\int_{0}^{\infty} 2^{-x} x^{1 / 2} d x=1+\left(\frac{\pi}{\log 2}\right)^{1 / 2} \leq 4
\end{aligned}
$$

Hence, by (S.15) and (S.16),

$$
\sum_{s=1}^{S} \eta_{s} \leq \sum_{s=1}^{S} \frac{12 C 2^{-s} R H^{1 / 2}\left(2^{-s} R, \mathbb{F}_{n}(R),\|\cdot\|_{n}\right)}{n^{1 / 2} \delta}+\sum_{s=1}^{S} \frac{2^{-s} s^{1 / 2}}{8} \leq \frac{1}{2}+\frac{1}{2}=1 .
$$

The definition of $\eta_{s}$ implies that

$$
H\left(2^{-s} R, \mathbb{F}_{n}(R),\|\underset{9}{\|}\|_{n}\right) \leq \frac{n \delta^{2} \eta_{s}^{2}}{144 C^{2} 2^{-2 s} R^{2}}
$$


which is used to bound the right side of (S.14) so that

$$
\sum_{s=1}^{S} 2 \exp \left\{2 H\left(2^{-s} R, \mathbb{F}_{n}(R),\|\cdot\|_{n}\right)-\frac{n \delta^{2} \eta_{s}^{2}}{36 C^{2} 2^{-2 s} R^{2}}\right\} \leq \sum_{s=1}^{S} 2 \exp \left\{-\frac{n \delta^{2} \eta_{s}^{2}}{72 C^{2} 2^{-2 s} R^{2}}\right\}
$$

Next, invoke that $\eta_{s} \geq 2^{-s} s^{1 / 2} / 8$ to get

$$
\begin{aligned}
& \sum_{s=1}^{S} 2 \exp \left\{-\frac{n \delta^{2} \eta_{s}^{2}}{72 C^{2} 2^{-2 s} R^{2}}\right\} \\
& \leq \sum_{s=1}^{S} 2 \exp \left\{-\frac{n \delta^{2} s}{4608 C^{2} R^{2}}\right\} \leq \sum_{s=1}^{\infty} 2 \exp \left\{-\frac{n \delta^{2} s}{4608 C^{2} R^{2}}\right\} \\
& =2 \exp \left\{-\frac{n \delta^{2}}{4608 C^{2} R^{2}}\right\}\left[1-\exp \left\{-\frac{n \delta^{2}}{4608 C^{2} R^{2}}\right\}\right]^{-1} \\
& \leq 4 \exp \left\{-\frac{n \delta^{2}}{4608 C^{2} R^{2}}\right\},
\end{aligned}
$$

where in the last inequality, we used (S.9) with a sufficiently large choice of $C_{0}$ to ensure that

$$
\frac{n^{1 / 2} \delta}{2} \geq(1152 \log 2)^{1 / 2} C R \quad \text { or } \quad \frac{n \delta^{2}}{4608 C^{2} R^{2}} \geq \log 2 .
$$

Recall that $C^{2}=8 r_{v}\left(M_{0}^{2}+\sigma_{0}^{2}\right)$. Hence we have proved (S.10) by setting $C_{0}^{2}=16 \mathrm{~V}$ $36846 r_{v}\left(M_{0}^{2}+\sigma_{0}^{2}\right) / 4$.

Lemma S.8. Suppose that $\mu( \pm v)$ increases slower than $v^{L}$ as $v \rightarrow \infty$ for some $L>0$. For some constant $M$ independent of $n$,

$$
N\left(r, \mathbb{F}_{n}(R),\|\cdot\|_{n}\right) \leq\left(\frac{M R^{L} Q_{n}^{L / 2}}{r}\right)^{2\left(D Q_{n}+K+1\right)} \text { a.s.. }
$$

Proof. We use $\mathbb{F}_{n}^{\mathrm{id}}$ and $\mathbb{F}_{n}^{\mathrm{id}}(R)$ to denote the special case of $\mathbb{F}$ and $\mathbb{F}_{n}(R)$ when $\mu(\cdot)$ is the identity link. By Example 3.7.4d on Page 40 of van de Geer (2000), we know that $\mathbb{F}_{n}^{\text {id }}$ is a $V C$ subgraph class with $V C$ index $V\left(\mathbb{F}_{n}^{\text {id }}\right) \leq D Q_{n}+K+2$. As a subset of $\mathbb{F}_{n}^{\text {id }}, \mathbb{F}_{n}^{\text {id }}(R)$ is also a $V C$ subgraph class with $V C$ index $V\left(\mathbb{F}_{n}^{\text {xid }}(R)\right) \leq D Q_{n}+K+2$. Since $\mu$ is a monotone function, by Lemma 2.6.18(viii) of van der Vaart and Wellner (1996), we have that $V\left(\mathbb{F}_{n}(R)\right) \leq V\left(\mathbb{F}_{n}^{\mathrm{id}}(R)\right) \leq D Q_{n}+K+2$. By the monotonicity of $\mu$, Lemmas S.2 and S.3, and the assumption on the growth rate of $\mu( \pm v)$ as $v \rightarrow \infty$, we have that

$$
\begin{aligned}
\|\mu(f)\|_{\infty} & \leq\left|\mu\left(\|f\|_{\infty}\right)\right|+\left|\mu\left(-\|f\|_{\infty}\right)\right| \\
& \leq\left|\mu\left(M_{3} \sqrt{Q_{n}} R\right)\right|+\left|\mu\left(-M_{3} \sqrt{Q_{n}} R\right)\right| \lesssim R^{L} Q_{n}^{L / 2},
\end{aligned}
$$


for any $\mu(f) \in \mathbb{F}_{n}(R)$, as $n \rightarrow \infty$. Applying Theorem 3.11 on Page of 40 of van de Geer (2000) with $\delta=r R^{-L} Q_{n}^{-L / 2}, p=2$, and $Q=\|\cdot\|_{n}$, we obtain that

$$
\begin{aligned}
N\left(r, \mathbb{F}_{n}(R),\|\cdot\|_{n}\right) & \leq C V\left(\mathbb{F}_{n}(R)\right)(16 e)^{V\left(\mathbb{F}_{n}(R)\right)}\left(\frac{R^{L} Q_{n}^{L / 2}}{r}\right)^{2\left\{V\left(\mathbb{F}_{n}(R)\right)-1\right\}} \\
& \leq C\left(D Q_{n}+K+2\right)(16 e)^{D Q_{n}+K+2}\left(\frac{R^{L} Q_{n}^{L / 2}}{r}\right)^{2\left(D Q_{n}+K+1\right)} \\
& \leq\left(\frac{M R^{L} Q_{n}^{L / 2}}{r}\right)^{2\left(D Q_{n}+K+1\right)}
\end{aligned}
$$

for some absolute constant $M$.

LEMMA S.9. Let

$$
J_{\delta}=\int_{\delta^{2} / 128 \sigma}^{\delta} H^{1 / 2}\left(u, \mathbb{F}_{n}(\delta),\|\cdot\|_{n}\right) d u \bigvee \delta, \quad 0<\delta<128 \sigma
$$

We have that

$$
n^{1 / 2} \delta^{2} \geq 32 C_{0} J_{\delta}
$$

for $\delta_{n} \leq \delta<128 \sigma$ and some $\delta_{n}$ satisfying $\left(Q_{n} / n\right)^{1 / 2} \leq \delta_{n} \leq\left(Q_{n} / n\right)^{1 / 2} \log n$.

Proof. By Lemma S.8 we have that

$$
J_{\delta} \leq \int_{\delta^{2} / 128 \sigma}^{\delta}\left\{2\left(D Q_{n}+K+1\right) \log \left(\frac{M \delta^{L} Q_{n}^{L / 2}}{u}\right)\right\}^{1 / 2} d u \bigvee \delta .
$$

Since $\delta<128 \sigma$ and the integrand is a decreasing function of $u$, we have

$$
J_{\delta} \leq \psi_{\delta}= \begin{cases}\delta\left\{2\left(D Q_{n}+K+1\right) \log \left(128 M \sigma \delta^{L-2} Q_{n}^{L / 2}\right)\right\}^{1 / 2} & \text { if } 0<L<2 ; \\ \delta\left\{2\left(D Q_{n}+K+1\right) \log \left(M(128 \sigma)^{L-1} Q_{n}^{L / 2}\right)\right\}^{1 / 2} & \text { if } L \geq 2 .\end{cases}
$$

Note that $\delta^{-2} \psi_{\delta}$ is a decreasing function of $\delta$ when $0<\delta<128 \sigma$. Thus,

$$
n^{1 / 2} \delta^{2} \geq 32 C_{0} \psi_{\delta}
$$

for all $\delta_{n}<\delta<128 \sigma$ with $\delta_{n}$ satisfies

$$
n^{1 / 2} \delta_{n}^{2}=32 C_{0} \psi_{\delta_{n}} .
$$

It is easy to verify that, for $\delta_{n 1}=\left(Q_{n} / n\right)^{1 / 2} \log n$,

$$
\delta_{n 1}^{-2} \psi_{\delta_{n 1}} \asymp \begin{cases}\frac{n^{1 / 2}}{\log n}\left[\log \left\{128 M \sigma\left(n / Q_{n}\right)^{1-L / 2}(\log n)^{L / 2-1} Q_{n}^{L / 2}\right\}\right]^{1 / 2} & \text { if } 0<L<2 ; \\ \frac{n^{1 / 2}}{\log n}\left[\log \left\{M(128 \sigma)^{L-1} Q_{n}^{L / 2}\right\}\right]^{1 / 2} & \text { if } L \geq 2\end{cases}
$$

Thus, $\delta_{n 1}^{-2} \psi_{\delta_{n 1}} \leq n^{1 / 2} /\left(32 C_{0}\right)$ for $n$ large. This implies that $\delta_{n} \leq \delta_{n 1}=\left(Q_{n} / n\right)^{1 / 2} \log n$, because $\delta^{-2} \psi_{\delta}$ is decreasing in $\delta$. Similarly it can be checked that, for $\delta_{n 2}=\left(Q_{n} / n\right)^{1 / 2}$, $\delta_{n 2}^{-2} \psi_{\delta_{n 2}} \geq n^{1 / 2} /\left(32 C_{0}\right)$ for $n$ large, which implies that $\delta_{n} \geq \delta_{n 2}=\left(Q_{n} / n\right)^{1 / 2}$. 


\section{S.4. Proof of Theorem 1}

Denote $\boldsymbol{W}_{i}=\boldsymbol{Y}_{i}-f_{n}^{*}\left(\mathbf{X}_{i}, \mathbf{T}_{i}\right)$. Then

$$
\boldsymbol{W}_{i}=\left\{\boldsymbol{Y}_{i}-f_{0}\left(\mathbf{X}_{i}, \mathbf{T}_{i}\right)\right\}+\left\{f_{0}\left(\mathbf{X}_{i}, \mathbf{T}_{i}\right)-f_{n}^{*}\left(\mathbf{X}_{i}, \mathbf{T}_{i}\right)\right\} \equiv \boldsymbol{e}_{i}+\boldsymbol{\xi}_{i} .
$$

In the following, $\boldsymbol{Y}_{i}, \boldsymbol{W}_{i}, \boldsymbol{e}_{i}$ and $\boldsymbol{\xi}_{i}$ will be viewed as evaluation of some functions, denoted as $y, w, e$ and $\xi$ respectively. For example, $y=y(\mathbf{x}, \mathbf{t})$ is a function that satisfies $y\left(\boldsymbol{X}_{i j}, \boldsymbol{T}_{i j}\right)=Y_{i j}$, and $w=w(\mathbf{x}, \mathbf{t})$ is a function that satisfies $w\left(\boldsymbol{X}_{i j}, \boldsymbol{T}_{i j}\right)=W_{i j}$. This functional viewpoint helps simplify our presentation. In particular, it facilitates the use of inner products and norms for representing some complicated expressions.

Recall the definition of the norm $\|\cdot\|_{n}$ given before the statement of Theorem 1 . Denote the corresponding inner product as $\langle\cdot, \cdot\rangle_{n}$. Since $w=y-f_{n}^{*}$ and

$$
\left\|y-f_{n}^{*}+f_{n}^{*}-\widehat{f}_{n}\right\|_{n}^{2}=\left\|y-\widehat{f}_{n}\right\|_{n}^{2} \leq\left\|y-f_{n}^{*}\right\|_{n}^{2},
$$

we have that

$$
\left\|\widehat{f}_{n}-f_{n}^{*}\right\|_{n}^{2} \leq 2\left\langle w, \widehat{f}_{n}-f_{n}^{*}\right\rangle_{n} .
$$

Let $\sigma$ be a positive constant to be determined later. It follows from (S.20) and the CauchySchwarz inequality $\left|\left\langle\varepsilon_{1}, \varepsilon_{2}\right\rangle_{n}\right| \leq\left\|\varepsilon_{1}\right\|_{n}\left\|\varepsilon_{2}\right\|_{n}$ that $\left\|\widehat{f}_{n}-f_{n}^{*}\right\|_{n} \leq 2 \sigma$ on $\left\{\|w\|_{n} \leq \sigma\right\}$. Let $\delta>0$ satisfy $\delta \leq 2 \sigma$. Define $S=\max \left\{s=0,1, \cdots: 2^{s} \delta<2 \sigma\right\}$. Applying the peeling device (see the proof of Theorem 9.1 of van de Geer, 2000), we obtain that

$$
\begin{aligned}
& P\left(\left\{\left\|\widehat{f}_{n}-f_{n}^{*}\right\|_{n}>\delta\right\} \cap\left\{\|w\|_{n} \leq \sigma\right\} \mid\left\{\mathbf{X}_{i}, \mathbf{T}_{i}\right\}_{i=1}^{n}\right) \\
& \leq \sum_{s=0}^{S} P\left(\left\{2^{s+1} \delta \geq\left\|\widehat{f}_{n}-f_{n}^{*}\right\|_{n}>2^{s} \delta\right\} \cap\left\{\|w\|_{n} \leq \sigma\right\} \mid\left\{\mathbf{X}_{i}, \mathbf{T}_{i}\right\}_{i=1}^{n}\right) \\
& \leq \sum_{s=0}^{S} P\left(\left\{\sup _{f \in \mathbb{F}_{n}\left(2^{s+1} \delta\right)} 2\left\langle w, f-f_{n}^{*}\right\rangle_{n}>2^{2 s} \delta^{2}\right\} \cap\left\{\|w\|_{n} \leq \sigma\right\} \mid\left\{\mathbf{X}_{i}, \mathbf{T}_{i}\right\}_{i=1}^{n}\right),
\end{aligned}
$$

where $\mathbb{F}_{n}(R)=\left\{f \in \mathbb{F}_{n}:\left\|f-f_{n}^{*}\right\|_{n} \leq R\right\}$, as defined earlier. Note that $\|\xi\|_{n}<c \rho_{n}$. If we set $\delta>8 c \rho_{n}$, then we have

$$
\left|\left\langle\xi, f-f_{n}^{*}\right\rangle_{n}\right| \leq c \rho_{n} \cdot 2^{s+1} \delta \leq 2^{2 s-2} \delta^{2}, \quad f \in \mathbb{F}_{n}\left(2^{s+1} \delta\right) .
$$

Notice the fact that $w=e+\xi$. The triangle inequality suggests that $\|e\|_{n} \leq 2 \sigma$ if $\|w\|_{n} \leq \sigma$ and $\sigma>c \rho_{n}$. Combining the above discussion, we have that

$$
\begin{aligned}
& P\left(\left\{\left\|\widehat{f}_{n}-f_{n}^{*}\right\|_{n}>\delta\right\} \cap\left\{\|w\|_{n} \leq \sigma\right\} \mid\left\{\mathbf{X}_{i}, \mathbf{T}_{i}\right\}_{i=1}^{n}\right) \\
& \leq \sum_{s=0}^{S} P\left(\left\{\sup _{f \in \mathbb{F}_{n}\left(2^{s+1} \delta\right)}\left|\left\langle e, f-f_{n}^{*}\right\rangle_{n}\right|>2^{2 s-2} \delta^{2}\right\}\right. \\
&\left.\cap\left\{\|e\|_{n} \leq 2 \sigma\right\} \mid\left\{\mathbf{X}_{i}, \mathbf{T}_{i}\right\}_{i=1}^{n}\right) .
\end{aligned}
$$


Note by Lemma S.9 that

$$
n^{1 / 2} 2^{2 s-2} \delta^{2} \geq 2 C_{0}\left\{\int_{2^{2 s-2} \delta^{2} / 8 \sigma}^{2^{s+1} \delta} H^{1 / 2}\left(u, \mathbb{F}_{n}\left(2^{s+1} \delta\right),\|\cdot\|_{n}\right) d u \bigvee 2^{s+1} \delta\right\},
$$

provided that $2^{s+1} \delta>\delta_{n}$ (to ensure this, assume WLOG $\delta>\delta_{n}$ ). Now we apply Lemma S.7. First, (S.9) is satisfied when $\delta$ and $R$ there are set to be $2^{2 s-2} \delta^{2}$ and $2^{s+1} \delta$, respectively. Then, by (S.10), we have

$$
\begin{gathered}
P\left(\left\{\sup _{f \in \mathbb{F}_{n}\left(2^{s+1} \delta\right)}\left|\left\langle e, f-f_{n}^{*}\right\rangle_{n}\right|>2^{2 s-2} \delta^{2}\right\} \cap\left\{\|e\|_{n} \leq 2 \sigma\right\} \mid\left\{\mathbf{X}_{i}, \mathbf{T}_{i}\right\}_{i=1}^{n}\right) \\
\leq C_{0} \exp \left(-\frac{n 2^{2 s} \delta^{2}}{256 C_{0}^{2}}\right) .
\end{gathered}
$$

It then follows from (S.21) that for $\delta \geq \delta_{n}+8 c \rho_{n}$,

$$
\begin{aligned}
& P\left(\left\{\left\|\widehat{f}_{n}-f_{n}^{*}\right\|_{n}>\delta\right\} \cap\left\{\|w\|_{n} \leq \sigma\right\} \mid\left\{\mathbf{X}_{i}, \mathbf{T}_{i}\right\}_{i=1}^{n}\right) \\
& \quad \leq \sum_{s=0}^{S} C_{0} \exp \left(-\frac{n 2^{2 s} \delta^{2}}{256 C_{0}^{2}}\right) \leq 2 C_{0} \exp \left(-\frac{n \delta^{2}}{256 C_{0}^{2}}\right) ;
\end{aligned}
$$

here, we use the inequality

$$
\sum_{s=0}^{\infty} \exp \left(-a 2^{2 s}\right) \leq \sum_{k=1}^{\infty} \exp (-a k)=\frac{e^{-a}}{1-e^{-a}} \leq 2 e^{-a},
$$

provided that $e^{-a}<1 / 2$. Therefore,

$$
\begin{aligned}
P\left(\left\|\widehat{f}_{n}-f_{n}^{*}\right\|_{n}>\delta \mid\left\{\mathbf{X}_{i}, \mathbf{T}_{i}\right\}_{i=1}^{n}\right) \leq & C_{0} \exp \left(-\frac{n \delta^{2}}{256 C_{0}^{2}}\right) \\
& +P\left(\|w\|_{n}>\sigma \mid\left\{\mathbf{X}_{i}, \mathbf{T}_{i}\right\}_{i=1}^{n}\right) .
\end{aligned}
$$

Note that we previously require $\delta \geq \delta_{n}+8 c \rho_{n}$. Then it follows that

$$
\begin{aligned}
& P\left(\left\|\widehat{f}_{n}-f_{n}^{*}\right\|_{n}>\delta_{n}+8 c \rho_{n}\right) \\
& \quad=E\left\{P\left(\left\|\widehat{f}_{n}-f_{n}^{*}\right\|_{n}>\delta_{n}+8 c \rho_{n} \mid\left\{\mathbf{X}_{i}, \mathbf{T}_{i}\right\}_{i=1}^{n}\right)\right\} \\
& \quad \leq 2 C_{0} \exp \left(-\frac{n \delta^{2}}{256 C_{0}^{2}}\right)+P\left(\|w\|_{n}>\sigma\right) .
\end{aligned}
$$

Let $c_{v}=\max _{i}\left\{\lambda_{\mathbf{v}_{i}^{-1}}^{\max }\right\}$, then $c_{v}<\infty$ by Condition C4. Set $\sigma=\|e\|_{n} / 2+c \max _{n} \rho_{n}+$ $\left(2 c_{v}\right)^{1 / 2} \sigma_{0}\left(\sigma_{0}\right.$ is defined in Condition C6). By the Chebyshev inequality,

$$
P\left(\|w\|_{n}>\sigma\right) \leq P\left(\|e\|_{n}>\left(2 c_{v}\right)^{1 / 2} 2 \sigma_{0}\right) \leq \exp \left(-\frac{8 n c_{v} \sigma_{0}^{2}}{M_{0}^{2}}\right) E\left(\exp \left(\frac{n\|e\|_{n}^{2}}{M_{0}^{2}}\right)\right) .
$$


According to Condition C6,

$$
E\left\{\exp \left(\frac{n\|e\|_{n}^{2}}{M_{0}^{2}}\right)\right\} \leq E\left\{\exp \left(\sum_{i=1}^{n} \frac{c_{v}\left|\boldsymbol{e}_{i}\right|^{2}}{M_{0}^{2}}\right)\right\} \leq\left(1+\frac{\sigma_{0}^{2}}{M_{0}^{2}}\right)^{n c_{v}} \leq \exp \left(\frac{n c_{v} \sigma_{0}^{2}}{M_{0}^{2}}\right) .
$$

Hence

$$
P\left(\|w\|_{n}>\sigma\right) \leq \exp \left(-\frac{7 n c_{v} \sigma_{0}^{2}}{M_{0}^{2}}\right) \rightarrow 0
$$

Consequently,

$$
P\left(\left\|\widehat{f}_{n}-f_{n}^{*}\right\|_{n}>\delta_{n}+8 c \rho_{n}\right) \rightarrow 0, \quad \text { as } n \rightarrow \infty .
$$

Since $\delta_{n} \leq\left(Q_{n} / n\right)^{1 / 2} \log n$, as required in application of Lemma S.9, the first conclusion follows.

By Condition C5(iii) and Lemma S.2, we have that

$$
\begin{aligned}
\left\|\widehat{f}_{n}-f_{n}^{*}\right\|_{\infty} & \lesssim\left\|\mathbf{x}^{\prime} \widehat{\boldsymbol{\beta}}_{V}+\widehat{\theta}-\left(\mathbf{x}^{\prime} \boldsymbol{\beta}_{0}+\theta_{n}^{*}\right)\right\|_{\infty} \\
& \lesssim Q_{n}^{1 / 2}\left\|\mathbf{x}^{\prime} \widehat{\boldsymbol{\beta}}_{V}+\widehat{\theta}-\left(\mathbf{x}^{\prime} \boldsymbol{\beta}_{0}+\theta_{n}^{*}\right)\right\| .
\end{aligned}
$$

Note that $\left(Q_{n} \log n\right)^{2} / n \rightarrow 0$. Moreover, $Q_{n} \rho_{n}^{2} \rightarrow 0$ by Condition C8 and the fact that $\rho_{n} \asymp Q_{n}^{-\alpha}$ for $\alpha>1 / 2$. It then follows by Condition C5(ii) and the first conclusion that

$$
\begin{aligned}
& Q_{n}^{1 / 2}\left\|\mathbf{x}^{\prime} \widehat{\boldsymbol{\beta}}_{V}+\widehat{\theta}-\left(\mathbf{x}^{\prime} \boldsymbol{\beta}_{0}+\theta_{n}^{*}\right)\right\| \\
& \quad \lesssim Q_{n}^{1 / 2}\left\|\widehat{f_{n}}-f_{n}^{*}\right\|=Q_{n}^{1 / 2} O_{P}\left\{\left(Q_{n} / n\right)^{1 / 2} \log n+\rho_{n}\right\}=o_{P}(1) .
\end{aligned}
$$

We thus obtain the second conclusion.

By Taylor's theorem,

$$
\begin{aligned}
& f_{n}^{*}(\mathbf{x}, t)-f_{0}(\mathbf{x}, t) \\
& \quad=\int_{0}^{1} \mu^{\prime}\left[\mathbf{x}^{T} \beta_{0}+\theta_{0,+}(t)+u\left\{\theta_{n}^{*}(t)-\theta_{0,+}(t)\right\}\right] d u\left\{\theta_{n}^{*}(t)-\theta_{0,+}(t)\right\} .
\end{aligned}
$$

Due to Condition C5(iii), it follows that $\left\|f_{n}^{*}-f_{0}\right\|_{\infty}=O\left(\left\|\theta_{n}^{*}-\theta_{0_{+}+}\right\|_{\infty}\right)=O\left(\rho_{n}\right)$. Combining this with the second conclusion gives $\left\|\widehat{f}_{n}-f_{0}\right\|_{\infty}=o_{P}(1)$, which is the third conclusion.

From the proof of the second conclusion, we have that

$$
\left\|\mathbf{x}^{\prime} \widehat{\boldsymbol{\beta}}_{V}+\widehat{\theta}-\left(\mathbf{x}^{\prime} \boldsymbol{\beta}_{0}+\theta_{n}^{*}\right)\right\|=O_{P}\left\{\left(Q_{n} / n\right)^{1 / 2} \log n+\rho_{n}\right\} .
$$

By Lemma 3.1 of Stone (1994),

$$
\left\|\mathbf{x}^{\prime}\left(\widehat{\boldsymbol{\beta}}_{V}-\boldsymbol{\beta}_{0}\right)\right\|^{2}+\left\|\widehat{\theta}-\theta_{n}^{*}\right\|^{2} \lesssim\left\|\mathbf{x}^{\prime} \widehat{\boldsymbol{\beta}}_{V}+\widehat{\theta}_{n}-\left(\mathbf{x}^{\prime} \boldsymbol{\beta}_{0}+\theta_{n}^{*}\right)\right\|^{2} .
$$


Combining these two results, we obtain that $\left\|\mathbf{x}^{\prime}\left(\widehat{\boldsymbol{\beta}}_{V}-\boldsymbol{\beta}_{0}\right)\right\|^{2}=o_{P}(1)$, which together with the no-multicollinearity condition C2 implies $\widehat{\boldsymbol{\beta}}_{V} \stackrel{P}{\rightarrow} \boldsymbol{\beta}_{0}$. We also obtain that $\left\|\widehat{\theta}-\theta_{n}^{*}\right\|=$ $O_{P}\left\{\left(Q_{n} / n\right)^{1 / 2} \log n+\rho_{n}\right\}$. Thus, by Lemma S.2,

$$
\left\|\widehat{\theta}-\theta_{n}^{*}\right\|_{\infty}=Q_{n}^{1 / 2} O_{P}\left\{\left(Q_{n} / n\right)^{1 / 2} \log n+\rho_{n}\right\}=o_{P}(1) .
$$

Since $\left\|\theta_{n}^{*}-\theta_{0,+}\right\|_{\infty}=O\left(\rho_{n}\right)=o(1)$, application of the triangle inequality yields $\| \widehat{\theta}-$ $\theta_{0,+} \|_{\infty}=o_{P}(1)$, the last conclusion.

The proof of the theorem is complete.

\section{S.5. Some Lemmas for Theorem 2}

The following Lemmas are needed for the asymptotic normality result Theorem 2 .

Lemma S.10. There exists a positive constant $M_{5}$ that is independent of $n$ such that

$$
\mid \mu^{\prime}\left(\boldsymbol{X}_{i j}^{\prime} \widehat{\boldsymbol{\beta}}_{V}+\boldsymbol{Z}_{i j}^{\prime} \widehat{\gamma}\right)-\mu^{\prime}\left(\boldsymbol{X}_{i j}^{\prime} \boldsymbol{\beta}_{0}+\theta_{0,+}\left(\boldsymbol{T}_{i j}\right)\left|\leq M_{5}\right| \widehat{d}_{i j} \mid,\right.
$$

where $\widehat{d}_{i j}$ is the jth element of $\widehat{\boldsymbol{d}}_{i}=\mathbf{X}_{i} \widehat{\boldsymbol{\beta}}_{V}+\mathbf{Z}_{i} \widehat{\boldsymbol{\gamma}}-\mathbf{X}_{i} \boldsymbol{\beta}_{0}-\theta_{0,+}\left(\mathbf{T}_{i}\right)$.

Proof. This is an immediate consequence of the intermediate value theorem, Theorem 1 , and Condition C5(iii).

Let $\widehat{\Pi}_{n}^{\Delta}, \Pi_{n}^{\Delta}$ denote respectively the projection onto $\mathbb{G}_{+}$relative to $\langle\cdot, \cdot\rangle_{n}^{\Delta}$ and $\langle\cdot, \cdot\rangle^{\Delta}$. Let $X_{i j k}$ denote the $k$-th component of $\boldsymbol{X}_{i j}$ and $\mathbf{H}_{21, k}$ denote the $k$-th column of $\mathbf{H}_{21}$ where $\mathbf{H}_{21}$ was defined in (20) of the main paper.

Lemma S.11. There exists a positive constant $M_{6}$ that is independent of $n$ such that,

$$
\max _{1 \leq i \leq n} \max _{1 \leq j \leq m_{i}}\left|X_{i j k}-\boldsymbol{Z}_{i j}^{\prime} \mathbf{H}_{22}^{-1} \mathbf{H}_{21, k}\right| \leq M_{6}, \quad k=1, \ldots, K,
$$

on $\Omega_{n}^{c}$ with $\Omega_{n}$ satisfying $P\left(\Omega_{n}\right) \rightarrow 0$.

Proof. Using the above defined inner product, we see that the least favorable direction $\varphi_{k,+}^{*}=\arg \min _{\varphi \in L_{2,+}}\left\|x_{k}-\varphi\right\|^{\Delta}$, where $L_{2,+}$ denote the space of square integrable additive functions. To avoid clutter, we drop the subscript + in the rest of the proof of this lemma. Define $\varphi_{k, n}^{*}=\Pi_{n}^{\Delta} x_{k}$ and $\widehat{\varphi}_{k, n}=\widehat{\Pi}_{n}^{\Delta} x_{k}$. Recall that $x_{k}(\cdot)$ denotes the coordinate mapping that maps $x$ to its $k$-th component. We know that $\left(x_{k}-\widehat{\Pi}_{n}^{\triangle} x_{k}\right)$ maps $X_{i j k}$ to $\left(X_{i j k}-\right.$ $\boldsymbol{Z}_{i j}^{\prime} \mathbf{H}_{22}^{-1} \mathbf{H}_{21, k}$ ). Thus, in order to show (S.24), we only need to show the boundedness of $\widehat{\varphi}_{k, n}$. We use the boundedness of $\varphi_{k}^{*}$ and $\varphi_{k, n}^{*}$ as a bridge.

In subsection S.1, we have already shown the the boundedness of $\varphi_{k}^{*}$. Now we show the the boundedness of $\varphi_{k, n}^{*}$. Let $g_{n}^{*} \in \mathbb{G}_{+}$be the minimizer of $\left\|\varphi_{k}^{*}-g\right\|_{\infty}$. Then we have $\left\|\varphi_{k}^{*}-g_{n}^{*}\right\|_{\infty} \lesssim \rho_{n}$. Since $\varphi_{k, n}^{*}-g_{n}^{*}=\Pi_{n}^{\Delta}\left(\varphi_{k}^{*}-g_{n}^{*}\right)$, we have

$$
\left\|\varphi_{k, n}^{*}-g_{n}^{*}\right\|^{\Delta} \leq\left\|\varphi_{k}^{*}-g_{n}^{*}\right\|^{\Delta} \lesssim \rho_{n} .
$$


It follows by applying Lemma S.2(ii) that $\left\|\varphi_{k, n}^{*}-g_{n}^{*}\right\|_{\infty} \lesssim Q_{n}^{1 / 2}\left\|\varphi_{k, n}^{*}-g_{n}^{*}\right\|^{\Delta} \lesssim Q_{n}^{1 / 2} \rho_{n}$ Hence,

$$
\begin{aligned}
\left\|\varphi_{k, n}^{*}\right\|_{\infty} & \leq\left\|\varphi_{k, n}^{*}-g_{n}^{*}\right\|_{\infty}+\left\|g_{n}^{*}-\varphi_{k}^{*}\right\|_{\infty}+\left\|\varphi_{k}^{*}\right\|_{\infty} \\
& \lesssim Q_{n}^{1 / 2} \rho_{n}+\rho_{n}+1 \lesssim 1 .
\end{aligned}
$$

Next we show the boundedness of $\widehat{\varphi}_{k, n}$. By the definition of $\widehat{\varphi}_{k, n}$ and $\varphi_{k, n}^{*}$ and the Cauchy-Schwarz inequality,

$$
\begin{aligned}
\left\|\widehat{\varphi}_{k, n}-\varphi_{k, n}^{*}\right\|_{n}^{\Delta} & =\sup _{g \in \mathbb{G}_{+}} \frac{\left|\left\langle\widehat{\varphi}_{k, n}-\varphi_{k, n}^{*}, g\right\rangle_{n}^{\Delta}\right|}{\|g\|_{n}^{\Delta}}=\sup _{g \in \mathbb{G}_{+}} \frac{\left|\left\langle x_{k}-\varphi_{k, n}^{*}, g\right\rangle_{n}^{\Delta}\right|}{\|g\|_{n}^{\Delta}} \\
& =\sup _{g \in \mathbb{G}_{+}} \frac{\left|\left\langle x_{k}-\varphi_{k, n}^{*}, g\right\rangle_{n}^{\Delta}-\left\langle x_{k}-\varphi_{k, n}^{*}, g\right\rangle^{\Delta}\right|}{\|g\|_{n}^{\Delta}} .
\end{aligned}
$$

By Lemma S.3, Lemma S.4 and the fact that $\left\|x_{k}-\varphi_{k, n}^{*}\right\|_{\infty}$ is bounded, i.e. (S.25), we have

$$
\begin{aligned}
& \sup _{g \in \mathbb{G}_{+}} \frac{\left|\left\langle x_{k}-\varphi_{k, n}^{*}, g\right\rangle_{n}^{\Delta}-\left\langle x_{k}-\varphi_{k, n}^{*}, g\right\rangle^{\Delta}\right|}{\|g\|_{n}^{\Delta}} \\
&=O_{P}\left(\sqrt{\frac{Q_{n}}{n}}\right)\left\|x_{k}-\varphi_{k, n}^{*}\right\|_{n}^{\Delta}=O_{P}\left(\sqrt{\frac{Q_{n}}{n}}\right) .
\end{aligned}
$$

Therefore, $\left\|\widehat{\varphi}_{k, n}-\varphi_{k, n}^{*}\right\|_{n}^{\Delta}=O_{P}\left(\left(Q_{n} / n\right)^{1 / 2}\right)$. Applying Lemma S.3, we have that

$$
\left\|\widehat{\varphi}_{k, n}-\varphi_{k, n}^{*}\right\|^{\Delta}=\left(1+o_{P}(1)\right)\left\|\widehat{\varphi}_{k, n}-\varphi_{k, n}^{*}\right\|_{n}^{\Delta}=O_{P}\left(\sqrt{\frac{Q_{n}}{n}}\right),
$$

which implies that

$$
\left\|\widehat{\varphi}_{k, n}-\varphi_{k, n}^{*}\right\|_{\infty} \leq \sqrt{Q_{n}}\left\|\widehat{\varphi}_{k, n}-\varphi_{k, n}^{*}\right\|^{\Delta}=O_{P}\left(\sqrt{\frac{Q_{n}^{2}}{n}}\right)=o_{P}(1) .
$$

Combining the triangular inequality with (S.25), we obtain that $\left\|\widehat{\varphi}_{k, n}\right\|_{\infty}$ is bounded with probability tending to one.

LEMMA S.12. $n \mathbf{H}^{11}=O_{P}(1)$.

Proof. The same argument as in the proof of Theorem 2 of Huang, Zhang and Zhou (2007) gives this result.

\section{S.6. Proof of Theorem 2}

Note that $\widehat{\boldsymbol{\beta}}_{V} \in \mathbb{R}^{K}$ and $\widehat{\gamma} \in \mathbb{R}^{Q_{n}}$ solve the estimating equations

$$
\left\{\begin{array}{c}
\sum_{i=1}^{n} \mathbf{X}_{i}^{\prime} \widehat{\Delta}_{i} \mathbf{V}_{i}^{-1}\left\{\boldsymbol{Y}_{i}-\mu\left(\mathbf{X}_{i} \widehat{\boldsymbol{\beta}}_{V}+\mathbf{Z}_{i} \widehat{\gamma}\right)\right\}=0 \\
\sum_{i=1}^{n} \mathbf{Z}_{i}^{\prime} \widehat{\boldsymbol{\Delta}}_{i} \mathbf{V}_{i}^{-1}\left\{\boldsymbol{Y}_{i}-\mu\left(\mathbf{X}_{i} \widehat{\boldsymbol{\beta}}_{V}+\mathbf{Z}_{i} \widehat{\gamma}\right)\right\}=0 \\
16
\end{array}\right.
$$


or, equivalently

$$
\sum_{i=1}^{n} \mathbf{U}_{i}^{\prime} \widehat{\boldsymbol{\Delta}}_{i} \mathbf{V}_{i}^{-1}\left\{\boldsymbol{Y}_{i}-\mu\left(\mathbf{X}_{i} \widehat{\boldsymbol{\beta}}_{V}+\mathbf{Z}_{i} \widehat{\gamma}\right)\right\}=0
$$

with $\mathbf{U}_{i}=\left(\mathbf{X}_{i}, \mathbf{Z}_{i}\right)$, and $\widehat{\boldsymbol{\Delta}}_{i}$ is a diagonal matrix with the diagonal elements being the first derivative of $\mu(\cdot)$ evaluated at $X_{i j}^{\prime} \widehat{\boldsymbol{\beta}}_{V}+Z_{i j}^{\prime} \widehat{\boldsymbol{\gamma}}, j=1, \ldots, m_{i}$. Recall that $\boldsymbol{\gamma}^{*}$ is assumed to satisfy $\rho_{n}=\left\|\theta_{0,+}-\boldsymbol{B}^{\prime} \boldsymbol{\gamma}^{*}\right\|_{\infty} \rightarrow 0$. Then the boundedness of $\mathbf{x}^{\prime} \boldsymbol{\beta}_{0}+\theta_{0}(\mathbf{t})$ implies the same property of $\mathbf{x}^{\prime} \boldsymbol{\beta}_{0}+\boldsymbol{B}^{\prime}(t) \boldsymbol{\gamma}^{*}$. By (17) in the main paper and Condition C5(ii), $\mathbf{x}^{\prime} \widehat{\boldsymbol{\beta}}_{V}+\boldsymbol{B}^{\prime}(t) \widehat{\boldsymbol{\gamma}}$ is also bounded.

Using the Taylor expansion, we have that

$$
\begin{aligned}
\mu\left(\mathbf{X}_{i} \widehat{\boldsymbol{\beta}}_{V}+\mathbf{Z}_{i} \widehat{\gamma}\right)=\mu & \left(\mathbf{X}_{i} \boldsymbol{\beta}_{0}+\theta_{0}\left(\boldsymbol{T}_{i}\right)\right) \\
& +\boldsymbol{\Delta}_{i 0}\left\{\mathbf{X}_{i}\left(\widehat{\boldsymbol{\beta}}_{V}-\boldsymbol{\beta}_{0}\right)+\mathbf{Z}_{i} \widehat{\gamma}-\theta_{0}\left(\boldsymbol{T}_{i}\right)\right\}+\widehat{\boldsymbol{r}}_{i}
\end{aligned}
$$

where $\widehat{\boldsymbol{r}}_{i}$ is a remainder term satisfying

$$
\widehat{\boldsymbol{r}}_{i} \lesssim \widehat{\boldsymbol{d}}_{i}^{\prime} \widehat{\boldsymbol{d}}_{i}
$$

with

$$
\widehat{\boldsymbol{d}}_{i}=\left(\widehat{d}_{i 1}, \ldots, \widehat{d}_{i m_{i}}\right)^{\prime}=\mathbf{X}_{i} \widehat{\boldsymbol{\beta}}_{V}+\mathbf{Z}_{i} \widehat{\gamma}-\mathbf{X}_{i} \boldsymbol{\beta}_{0}-\theta_{0}\left(\boldsymbol{T}_{i}\right), \quad i=1, \cdots, n .
$$

Note that the second line of (S.27) can be rewritten as

$$
\boldsymbol{\Delta}_{i 0} \mathbf{U}_{i}\left(\begin{array}{c}
\widehat{\boldsymbol{\beta}}_{V}-\boldsymbol{\beta}_{0} \\
\widehat{\gamma}-\boldsymbol{\gamma}^{*}
\end{array}\right)+\boldsymbol{\Delta}_{i 0}\left(\mathbf{Z}_{i} \boldsymbol{\gamma}^{*}-\theta_{0}\left(\boldsymbol{T}_{i}\right)\right)+\widehat{\boldsymbol{r}}_{i}
$$

Define

$$
\widetilde{\boldsymbol{J}}_{1}=\left(\widehat{\boldsymbol{\Delta}}_{i}-\boldsymbol{\Delta}_{i 0}\right) \mathbf{V}_{i}^{-1}\left\{\boldsymbol{Y}_{i}-\mu\left(\mathbf{X}_{i} \widehat{\boldsymbol{\beta}}_{V}+\mathbf{Z}_{i} \widehat{\gamma}\right)\right\}
$$

and

$$
\boldsymbol{J}_{2}=\boldsymbol{\Delta}_{i 0} \mathbf{V}_{i}^{-1}\left\{\boldsymbol{Y}_{i}-\mu\left(\mathbf{X}_{i} \boldsymbol{\beta}_{0}+\theta_{0}\left(\boldsymbol{T}_{i}\right)\right)-\widehat{\boldsymbol{r}}_{i}-\boldsymbol{\Delta}_{i 0}\left(\mathbf{Z}_{i} \boldsymbol{\gamma}^{*}-\theta_{0}\left(\boldsymbol{T}_{i}\right)\right)\right\} .
$$

Substituting (S.27) into (S.26) yields

$$
0=\sum_{i=1}^{n} \mathbf{U}_{i}^{\prime}\left(\boldsymbol{J}_{1}+\boldsymbol{J}_{2}\right)-\sum_{i=1}^{n} \mathbf{U}_{i}^{\prime} \boldsymbol{\Delta}_{i 0} \mathbf{V}_{i}^{-1} \boldsymbol{\Delta}_{i 0} \mathbf{U}_{i}\left(\begin{array}{c}
\widehat{\boldsymbol{\beta}}_{V}-\boldsymbol{\beta}_{0} \\
\widehat{\gamma}-\boldsymbol{\gamma}^{*}
\end{array}\right) .
$$

Recalling (20) and using (21) in the main paper, we obtain from (S.30) that

$$
\widehat{\boldsymbol{\beta}}_{V}=\boldsymbol{\beta}_{0}+\mathbf{H}^{11} \sum_{i=1}^{n}\left(\mathbf{X}_{i}-\mathbf{Z}_{i} \mathbf{H}_{22}^{-1} \mathbf{H}_{21}\right)^{\prime}\left(\widetilde{\boldsymbol{J}}_{1}+\boldsymbol{J}_{2}\right) .
$$


Recall that $\boldsymbol{e}_{i}=\boldsymbol{Y}_{i}-\mu\left(\mathbf{X}_{i} \boldsymbol{\beta}_{0}+\theta_{0}\left(\boldsymbol{T}_{i}\right)\right)$ and define $\boldsymbol{\zeta}_{i}=\mu\left(\mathbf{X}_{i} \boldsymbol{\beta}_{0}+\theta_{0}\left(\boldsymbol{T}_{i}\right)\right)-\mu\left(\mathbf{X}_{i} \widehat{\boldsymbol{\beta}}_{V}+\mathbf{Z}_{i} \widehat{\gamma}\right)$. Define

$$
\begin{aligned}
\boldsymbol{I}_{1} & =\mathbf{H}^{11} \sum_{i=1}^{n}\left(\mathbf{X}_{i}-\mathbf{Z}_{i} \mathbf{H}_{22}^{-1} \mathbf{H}_{21}\right)^{\prime}\left(\widehat{\boldsymbol{\Delta}}_{i}-\boldsymbol{\Delta}_{i 0}\right) \mathbf{V}_{i}^{-1} \boldsymbol{e}_{i} \\
\boldsymbol{I}_{2} & =\mathbf{H}^{11} \sum_{i=1}^{n}\left(\mathbf{X}_{i}-\mathbf{Z}_{i} \mathbf{H}_{22}^{-1} \mathbf{H}_{21}\right)^{\prime}\left(\widehat{\boldsymbol{\Delta}}_{i}-\boldsymbol{\Delta}_{i 0}\right) \mathbf{V}_{i}^{-1} \boldsymbol{\zeta}_{i} \\
\boldsymbol{I}_{3} & =\mathbf{H}^{11} \sum_{i=1}^{n}\left(\mathbf{X}_{i}-\mathbf{Z}_{i} \mathbf{H}_{22}^{-1} \mathbf{H}_{21}\right)^{\prime} \boldsymbol{\Delta}_{i 0} \mathbf{V}_{i}^{-1} \boldsymbol{e}_{i} \\
\boldsymbol{I}_{4} & =\mathbf{H}^{11} \sum_{i=1}^{n}\left(\mathbf{X}_{i}-\mathbf{Z}_{i} \mathbf{H}_{22}^{-1} \mathbf{H}_{21}\right)^{\prime} \boldsymbol{\Delta}_{i 0} \mathbf{V}_{i}^{-1} \widehat{\boldsymbol{r}}_{i} \\
\boldsymbol{I}_{5} & =\mathbf{H}^{11} \sum_{i=1}^{n}\left(\mathbf{X}_{i}-\mathbf{Z}_{i} \mathbf{H}_{22}^{-1} \mathbf{H}_{21}\right)^{\prime} \boldsymbol{\Delta}_{i 0} \mathbf{V}_{i}^{-1} \boldsymbol{\Delta}_{i 0}\left\{\mathbf{Z}_{i} \boldsymbol{\gamma}^{*}-\theta_{0}\left(\boldsymbol{T}_{i}\right)\right\}
\end{aligned}
$$

Then, (S.31) can be written as

$$
\widehat{\boldsymbol{\beta}}_{V}=\boldsymbol{\beta}_{0}+\boldsymbol{I}_{1}+\boldsymbol{I}_{2}+\boldsymbol{I}_{3}-\boldsymbol{I}_{4}-\boldsymbol{I}_{5}
$$

To prove the asymptotic linear expansion (22) in the main paper, we need to show that

$$
\left|\boldsymbol{I}_{1}\right|+\left|\boldsymbol{I}_{2}\right|+\left|\boldsymbol{I}_{4}\right|+\left|\boldsymbol{I}_{5}\right|=o_{P}\left(n^{-1 / 2}\right) .
$$

Write $\boldsymbol{I}_{1}=n \mathbf{H}^{11} \boldsymbol{I}_{1}^{o}$, where

$$
\boldsymbol{I}_{1}^{o}=\frac{1}{n} \sum_{i=1}^{n}\left(\mathbf{X}_{i}-\mathbf{Z}_{i} \mathbf{H}_{22}^{-1} \mathbf{H}_{21}\right)^{\prime}\left(\widehat{\boldsymbol{\Delta}}_{i}-\boldsymbol{\Delta}_{i 0}\right) \mathbf{V}_{i}^{-1} \boldsymbol{e}_{i}
$$

Set

$$
h_{k}(\mathbf{x}, \mathbf{t} ; \boldsymbol{\beta}, \boldsymbol{\gamma})=\left\{\mu^{\prime}\left(\mathbf{x}^{\prime} \boldsymbol{\beta}+\boldsymbol{B}^{\prime}(\mathbf{t}) \boldsymbol{\gamma}\right)-\mu^{\prime}\left(\mathbf{x}^{\prime} \boldsymbol{\beta}_{0}+\theta_{0,+}(\mathbf{t})\right)\right\}\left\{x_{k}-\boldsymbol{B}(\mathbf{t})^{\prime} \mathbf{H}_{22}^{-1} \mathbf{H}_{21, k}\right\},
$$

where $x_{k}$ is the $k$-th element of $\mathbf{x}$ and $\mathbf{H}_{21, k}$ is the $k$-th column of $\mathbf{H}_{21}$. Then the $k$-th element of $\boldsymbol{I}_{1}^{o}$ is

$$
\left[\boldsymbol{I}_{1}^{o}\right]_{k}=\frac{1}{n} \sum_{i=1}^{n} h_{k}^{\prime}\left(\mathbf{X}_{i}, \mathbf{T}_{i} ; \widehat{\boldsymbol{\beta}}_{V}, \widehat{\gamma}\right) \mathbf{V}_{i}^{-1} \boldsymbol{e}_{i}
$$

for $k=1,2, \ldots, K$. Note that $\widehat{f}(\mathbf{x}, \mathbf{t})=\mu\left(\mathbf{x}^{\prime} \widehat{\boldsymbol{\beta}}_{V}+B^{\prime}(\mathbf{t}) \widehat{\gamma}\right)$ and $f_{0}(\mathbf{x}, \mathbf{t})=\mu\left(\mathbf{x}^{\prime} \boldsymbol{\beta}_{0}+\theta_{0,+}(\mathbf{t})\right)$. For notational simplicity, we write $h_{k}(\cdot)$ as $h(\cdot)$ hereafter. By Lemmas S.10 and S.11, Condition C5, and (S.22), the following holds on $\Omega_{n}^{c}$ with $P\left(\Omega_{n}\right) \rightarrow 0$,

$$
\left\|h\left(\cdot ; \widehat{\boldsymbol{\beta}}_{V}, \widehat{\gamma}\right)\right\|_{n} \leq c_{1}\left\|\widehat{f}_{n}-f_{0}\right\|_{n} \leq c_{1}\left\|\widehat{f}_{n}-f_{18}^{*}\right\|_{n}+c_{1}\left\|f_{n}^{*}-f_{0}\right\|_{n} \leq c_{1}\left(\delta_{n}+\rho_{n}\right) \equiv \tau_{n} .
$$


Recall that $\mathbb{F}_{n}^{\text {id }}=\left\{f(\mathbf{x}, \mathbf{t})=\mathbf{x}^{\prime} \boldsymbol{\beta}+g(\mathbf{t}): \boldsymbol{\beta} \in \mathbb{R}^{K}, g \in \mathbb{G}_{+}\right\}$. Let

$$
\begin{aligned}
\mathbb{H}_{n}=\{h(\mathbf{x}, \mathbf{t} ; f): h(\mathbf{x}, \mathbf{t} ; f)= \\
{\left.\left[\mu^{\prime}(f(\mathbf{x}, \mathbf{t}))-\mu^{\prime}\left(f_{0}(\mathbf{x}, \mathbf{t})\right)\right]\left[x_{k}-\boldsymbol{B}(\mathbf{t})^{\prime} \mathbf{H}_{22}^{-1} \mathbf{H}_{21, k}\right], f \in \mathbb{F}_{n}^{\mathrm{id}}\right\} }
\end{aligned}
$$

and $\mathbb{H}_{n}(R)=\left\{h \in \mathbb{H}_{n}:\|h\|_{n} \leq R\right\}$. For $f_{1}, f_{2} \in \mathbb{F}_{n}^{\text {id }}$, let $h_{1}(\cdot)=h\left(\cdot ; f_{1}\right)$ and $h_{2}(\cdot)=$ $h\left(\cdot ; f_{2}\right) \in \mathbb{H}_{n}$. It follows from Lemmas S.10 and S.11 that $\left\|h_{1}-h_{2}\right\|_{n} \leq c_{2}\left\|f_{1}-f_{2}\right\|_{n}$ for some constant $c_{2}>0$. Thus,

$$
N\left(2 c_{2} u, \mathbb{H}_{n}\left(\tau_{n}\right),\|\cdot\|_{n}\right) \leq N\left(u, \mathbb{F}_{n}^{\mathrm{id}}\left(\tau_{n}\right),\|\cdot\|_{n}\right) \leq\left(\frac{4 \tau_{n}+u}{u}\right)^{Q_{n}+K}
$$

by Lemma 2.5 on Page 20 of van de Geer (2000). We have that, on $\Omega_{n}^{c}$,

$$
\left|\left[\boldsymbol{I}_{1}^{o}\right]_{k}\right| \leq \sup _{h \in \mathbb{H}_{n}\left(\tau_{n}\right)} n^{-1}\left|\sum_{i=1}^{n} h\left(\mathbf{X}_{i}, \boldsymbol{T}_{i}\right)^{\prime} \mathbf{V}_{i}^{-1} \boldsymbol{e}_{i}\right| \equiv \sup _{h \in \mathbb{H}_{n}\left(\tau_{n}\right)}\left|\widetilde{\boldsymbol{I}}_{1}\right| .
$$

Note that for some large $\sigma,\|e\|_{n}<\sigma$ with probability tending to one. Now we apply Lemma S.7 with $R=\tau_{n}$ and $\sigma=2\|e\|_{n}$. Note that (S.9) holds for $\delta=c_{3} \tau_{n}\left(Q_{n} \log n / n\right)^{1 / 2}$. We have by (S.10) that, on $\Omega_{n}^{c}$,

$$
\begin{gathered}
P\left(\left\{\sup _{h \in \mathbb{H}_{n}\left(\tau_{n}\right)}\left|\widetilde{\boldsymbol{I}}_{1}\right| \geq c_{3} \tau_{n}\left(\frac{Q_{n} \log n}{n}\right)^{1 / 2}\right\} \cap\left\{\|e\|_{n} \leq \sigma\right\} \mid\left\{\mathbf{X}_{i}, \mathbf{T}_{i}\right\}_{i=1}^{n}\right) \\
\leq C_{0} \exp \left(-\frac{c_{3}^{2} Q_{n} \log n}{4 C_{0}^{2}}\right) .
\end{gathered}
$$

Therefore,

$$
\begin{aligned}
P\left(\left|\left[\boldsymbol{I}_{1}^{o}\right]_{k}\right|\right. & \left.\geq c_{3} \tau_{n}\left(\frac{Q_{n} \log n}{n}\right)^{1 / 2}\right) \\
& \leq C_{0} \exp \left(-\frac{c_{3}^{2} Q_{n} \log n}{4 C_{0}^{2}}\right)+P\left(\Omega_{n}\right) \rightarrow 0 \quad \text { as } n \rightarrow \infty
\end{aligned}
$$

This together with Lemma S.12 and Condition C8 implies that

$$
\boldsymbol{I}_{1}=\left(n \mathbf{H}^{11}\right) \boldsymbol{I}_{1}^{o}=O_{P}(1) O_{P}\left(c_{3} \tau_{n}\left(\frac{Q_{n} \log n}{n}\right)^{1 / 2}\right)=o_{P}\left(n^{-1 / 2}\right) .
$$

By the Cauchy-Schwarz inequality, Condition C4, Lemmas S.10, S.11 and S.12, and then by Theorem 1 and Condition C8,

$$
\left|\boldsymbol{I}_{2}\right|=O_{P}(1)\left(\frac{1}{n} \sum_{i=1}^{n} \sum_{j=1}^{m_{i}} \widehat{d}_{i j}^{2}\right)^{1 / 2}\left(\frac{1}{n} \sum_{i=1}^{n} \sum_{j=1}^{m_{i}} \zeta_{i j}^{2}\right)^{1 / 2}
$$




$$
=O_{P}\left(\sqrt{\frac{Q_{n}}{n}} \log n+\rho_{n}\right)^{2}=o_{P}\left(n^{-1 / 2}\right) .
$$

It follows from Lemma S.11, Conditions C4 and C5(ii), inequality (S.28), and then from Theorem 1 that

$$
\left|\boldsymbol{I}_{4}\right|=O_{P}(1) \frac{1}{n} \sum_{i=1}^{n} \sum_{j=1}^{m_{i}} \widehat{d}_{i j}^{2}=O_{P}\left(\sqrt{\frac{Q_{n}}{n}} \log n+\rho_{n}\right)^{2}=o_{P}\left(n^{-1 / 2}\right) .
$$

We now give an upper bound for $\boldsymbol{I}_{5}$. We show that the same argument as in dealing with the bias term for the identity link case (Huang, Zhang and Zhou, 2007) can be used for our purposes. Denote $\delta(\mathbf{t})=\boldsymbol{B}^{\prime}(\mathbf{t}) \boldsymbol{\gamma}^{*}-\theta_{0}(\mathbf{t})$ and $\boldsymbol{\delta}_{i}=\delta\left(\mathbf{T}_{i}\right)$. We have that

$$
\boldsymbol{I}_{5}=\mathbf{H}^{11} \sum_{i=1}^{n}\left(\mathbf{X}_{i}-\mathbf{Z}_{i} \mathbf{H}_{22}^{-1} \mathbf{H}_{21}\right)^{\prime} \boldsymbol{\Delta}_{i 0} \mathbf{V}_{i}^{-1} \boldsymbol{\Delta}_{i 0} \boldsymbol{\delta}_{i} \equiv n \mathbf{H}^{11} \boldsymbol{S}
$$

where $\boldsymbol{S}=\left(S_{1}, \cdots, S_{K}\right)^{\prime}$ and

$$
S_{k}=\left\langle x_{k}-\widehat{\Pi}_{n}^{\Delta} x_{k}, \delta\right\rangle_{n}^{\Delta}=\left\langle x_{k}-\widehat{\Pi}_{n}^{\Delta} x_{k}, \delta-\widehat{\Pi}_{n}^{\Delta} \delta\right\rangle_{n}^{\Delta}=\left\langle x_{k}, \delta-\widehat{\Pi}_{n}^{\Delta} \delta\right\rangle_{n}^{\Delta}
$$

Observe that $\|\delta(\cdot)\|_{\infty} \leq \rho_{n}$. It follows from exactly the same argument as in the proof of Theorem 2 of Huang, Zhang and Zhou (2007) that $\left|S_{k}\right|=o_{P}\left(n^{-1 / 2}\right)$. By Lemma S.12, $n \mathbf{H}^{11}=O_{P}(1)$ and as a consequence, $\boldsymbol{I}_{5}=o_{P}\left(n^{-1 / 2}\right)$. The proof of (22) in the main paper is complete.

By Lemma S.11, Lemma S.12, Conditions C3, C4, and C5(iii), we have

$$
\mathbf{R}^{\triangle}\left(\widehat{\boldsymbol{\beta}}_{V}\right)=O_{P}\left(\frac{1}{n}\right)
$$

Then, we have, by the asymptotic linear expansion proven above,

$$
\begin{aligned}
\left\{\mathbf{R}^{\triangle}\left(\widehat{\boldsymbol{\beta}}_{V}\right)\right\}^{-1 / 2}\left(\widehat{\boldsymbol{\beta}}_{V}-\boldsymbol{\beta}_{0}\right) & \\
& =\left\{\mathbf{R}^{\triangle}\left(\widehat{\boldsymbol{\beta}}_{V}\right)\right\}^{-1 / 2}\left(\mathbf{H}^{11} \sum_{i=1}^{n}\left(\mathbf{X}_{i}-\mathbf{Z}_{i} \mathbf{H}_{22}^{-1} \mathbf{H}_{21}\right)^{\prime} \boldsymbol{\Delta}_{i 0} \mathbf{V}_{i}^{-1} \boldsymbol{e}_{i}\right)+o_{P}(1) .
\end{aligned}
$$

Then by applying the central limit theorem to the above equation and using the fact that

$$
\operatorname{var}\left(\mathbf{H}^{11} \sum_{i=1}^{n}\left(\mathbf{X}_{i}-\mathbf{Z}_{i} \mathbf{H}_{22}^{-1} \mathbf{H}_{21}\right)^{\prime} \boldsymbol{\Delta}_{i 0} \mathbf{V}_{i}^{-1} \boldsymbol{e}_{i} \mid\left\{\mathbf{X}_{i}, \mathbf{T}_{i}\right\}_{i=1}^{n}\right)=\mathbf{R}^{\Delta}\left(\widehat{\boldsymbol{\beta}}_{V}\right)
$$

we obtain the second conclusion. This completes the whole proof. 


\section{S.7. More Simulation Results}

We have ran a simulation study to compare our method with that by Carroll et al. (2009) under exactly the same setups used in their paper. The results are reported in Table S1. We found that our spline method performs comparably to the local polynomial methods of Carroll et al. (2009) in these simple settings.

TABLE S1

MSEs $\times 10^{4}$ of the estimates for the Euclidean parameter based on 500 samples of size 200 on the exact same setups as Carroll et al. (2009). The methods "Local constant", "Local linear", and our spline method are labeled as $L C, L L$, and SP, respectively. Results for $L C, L L$ are adapted from Carroll, et al. (2009), Table 1.

\begin{tabular}{ccccccc}
\hline Setup & \multicolumn{2}{c}{ Accounting for correlation } & \multicolumn{3}{c}{ Working independence } \\
& LC & LL & SP & LC & LL & SP \\
\hline 1 & 2 & 2 & 1 & 12 & 12 & 7 \\
2 & 9 & 9 & 4 & 13 & 13 & 7 \\
3 & 15 & 16 & 6 & 16 & 16 & 6 \\
4 & 4 & 4 & 3 & 14 & 14 & 8 \\
5 & 2 & 3 & 1 & 14 & 14 & 6 \\
6 & 6 & 6 & 12 & 62 & 63 & 29 \\
7 & 31 & 31 & 27 & 67 & 67 & 28 \\
\hline
\end{tabular}

\section{References}

Bickel, P. J., Klaassen, A. J., Ritov, Y. and Wellner, J. A. (1993). Efficient and Adaptive Estimation for Semiparametric Models. Johns Hopkins University Press, Baltimore.

Carroll, R. J., Maity, A., Mammen, E. and Yu, K. (2009). Efficient semiparametric marginal Estimation for partially linear additive model for longitudinal/clustered data. Statistics in BioSciences 1 10-31.

HuAng, J. Z. (1998). Projection estimation in multiple regression with application to functional ANOVA models. Annals of Statistics 26 242-272.

HuANG, J. Z. (2003). Local asymptotics for polynomial spline regression. Annals of Statistics 31 1600-1635.

Huang, J. Z., Wu, C. O. and Zhou, L. (2004). Polynomial spline estimation and inference for varying coefficient models with longitudinal data. Statistica Sinica 14 763788.

HuAng, J. Z., Zhang, L. and Zhou, L. (2007). Efficient estimation in marginal partially linear models for longitudinal/clustered data using splines. Scandinavian Journal of Statistics 34 451-477. 
Kress, R. (1999). Linear Integral Equations, Second ed. Springer, New York.

Stone, C. J. (1994). The use of polynomial splines and their tensor products in multivariate function estimation. Annals of Statistics 22 118-171.

VAN DE Geer, S. (2000). Empirical Processes in M-Estimation. Cambridge University Press.

VAn der VaArt, A. W. and Wellner, J. A. (1996). Weak Convergence and Empirical Processes: With Applications to Statistics. Springer, New York. 